\title{
NLO QCD corrections to Single Top and W associated photoproduction at the LHC with forward detector acceptances
}

\author{
Hao Sun, ${ }^{a}$ Wei Liu, ${ }^{a}$ Xiao-Juan Wang, ${ }^{a}$ Ya-Jin Zhou ${ }^{b}$ and Hong-Sheng $\mathrm{Hou}^{c}$ \\ ${ }^{a}$ Institute of Theoretical Physics, School of Physics 85 Optoelectronic Technology, \\ Dalian University of Technology, \\ No. 2 Linggong Road, Dalian, Liaoning, 116024 P.R. China \\ ${ }^{b}$ School of Physics, Shandong University, \\ No. 27 South Shandong University Road, Jinan, Shandong, 250100 P.R. China \\ ${ }^{c}$ Department of Physics, Hangzhou Normal University, \\ No. 58 Haishu Road, Hangzhou, Zhejiang, 310036 P.R. China \\ E-mail: haosun@mail.ustc.edu.cn, liusd12@mail.dlut.edu.cn, \\ wangxiaojuan@mail.dlut.edu.cn, zhouyj@sdu.edu.cn, hshou@hznu.edu.cn
}

ABSTRACT: In this paper we study the Single Top and W boson associated photoproduction via the main reaction $\mathrm{pp} \rightarrow \mathrm{p} \gamma \mathrm{p} \rightarrow \mathrm{pW}^{ \pm} \mathrm{t}+\mathrm{Y}$ at the $14 \mathrm{TeV}$ Large Hadron Collider (LHC) up to next-to-leading order (NLO) QCD level assuming a typical LHC multipurpose forward detector. We use the Five-Flavor-Number Schemes (5FNS) with massless bottom quark assumption in the whole calculation. Our results show that the QCD NLO corrections can reduce the scale uncertainty. The typical K-factors are in the range of 1.15 to 1.2 which lead to the QCD NLO corrections of $15 \%$ to $20 \%$ correspond to the leading-order (LO) predictions with our chosen parameters.

KEYWORDS: NLO Computations

ARXIV EPRINT: 1408.1218 


\section{Contents}

1 Introduction 1

2 Calculation framework 4

2.1 Equivalent photon approximation 4

2.2 General $\gamma$ p photoproduction cross section 5

$\begin{array}{lll}2.3 & \text { Wt photoproduction at leading order } & 6\end{array}$

$\begin{array}{lll}2.4 & \text { Wt photoproduction at QCD next-to-leading order } & 7\end{array}$

$\begin{array}{lll}2.4 .1 & \text { General description } & 7\end{array}$

2.4.2 Virtual 8

$\begin{array}{lll}2.4 .3 & \text { Parton radiation } & 9\end{array}$

$\begin{array}{ll}\text { 2.4.4 Total QCD NLO cross section } & 12\end{array}$

3 Numerical results $\quad 13$

$\begin{array}{lll}3.1 & \text { Input parameters } & 13\end{array}$

$\begin{array}{lll}3.2 & \text { Cross checks } & 13\end{array}$

3.3 Scale dependence for different forward detector acceptances 14

$\begin{array}{lll}3.4 & \text { Distribution and cross section } & 17\end{array}$

$\begin{array}{llr}4 & \text { Summary } & 21\end{array}$

\section{Introduction}

The Large Hadron Collider (LHC) at CERN generates high energetic proton-proton (pp) collisions with a luminosity of $\mathcal{L}=10^{34} \mathrm{~cm}^{-2} \mathrm{~s}^{-1}$ and provides the opportunity to study very high energy physics. After the discovery of Higgs boson [1, 2], probing new physics beyond the Standard Model (BSM) turns to the main goal of the LHC. In such context, studying the heaviest elementary particle, the top quark, is particularly interesting since it is the only fermion with a natural Yukawa coupling to the Higgs boson of the order of unity. Its charged weak coupling might be sensitive to the existence of an additional heavy fermion. These couplings can be probed by measuring specific top quark production cross sections and branching ratios. However, these measurements will be challenging due to the composite internal structure of the colliding particles, i.e., the large QCD or electroweak (EW) backgrounds, the unknown precise centre-of-mass (c.m.s.) energy of the collisions occurring between the partons of protons, the complicate composition of underlying events within the central detector, etc. In this case, very high energy interactions involving quasireal incoming photons may provide a solution to some of these problems.

General diagrams for the photon induced interactions at the LHC is presented in figure 1. $\mathrm{pp} \rightarrow \mathrm{p} \gamma \gamma \mathrm{p} \rightarrow \mathrm{pXp}$ [left figure] refers to the photon-photon $(\gamma \gamma)$ interaction where 

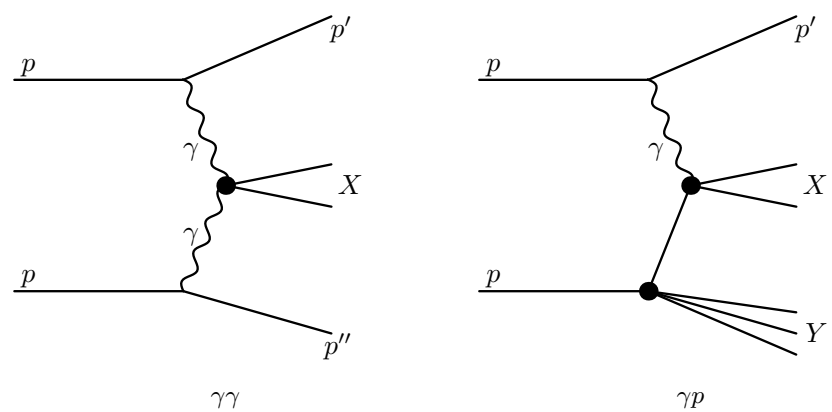

Figure 1. Generic diagrams for the photon induced production at the CERN LHC: pp $\rightarrow \mathrm{p} \gamma \gamma \mathrm{p} \rightarrow$ $\mathrm{pXp}$ [left figure] and $\mathrm{pp} \rightarrow \mathrm{p} \gamma \mathrm{p} \rightarrow \mathrm{pXY}$ [right figure].

photons radiated off by both protons collide and produce a central system $\mathrm{X}$. The system $\mathrm{X}$ will be detected by the central detector under clean experimental conditions and the two protons remain intact (namely forward protons), escape from the central detection and continue their path close to the beam line. $\mathrm{pp} \rightarrow \mathrm{p} \gamma \mathrm{p} \rightarrow \mathrm{pXY}$ [right figure] corresponds to photoproduction or photon-proton $(\gamma \mathrm{p})$ production: a photon from a proton induces a deep inelastic scattering with the incoming proton and produces a proton remnant $\mathrm{Y}$ in addition to the centrally produced $\mathrm{X}$ system. Despite a lower available luminosity, photoproduction can occur under better known initial conditions, with fewer final states particles and at high energy scale $(\sim \mathrm{TeV})$, thus can be studied as a complementary tool to normal pp collisions at the LHC. Indeed, the CDF collaboration has already observed such kinds of phenomenon including the exclusive dilepton [3, 4], diphoton [5, 6], dijet [7] production and charmonium $(\mathrm{J} / \psi)$ meson photoproduction [8], etc. Both the ATLAS and the CMS collaborations have programs of forward physics. They are devoted to studies of high rapidity regions with extra updated detectors located in a place nearly $100-400 \mathrm{~m}$ close to the interaction point [9-14]. Technical details of the ATLAS Forward Physics (AFP) projects can be found, for example, in refs. [15, 16]. A brief review of experimental prospects for studying photon induced interactions are summarized in ref. [17].

As previously mentioned, the top quark is the heaviest known elementary particle which makes it an excellent candidate for new physics searches. Among top quark production channels, Single Top production has some special features that top pair production can not achieve: it offers a unique possibility of the direct measurement of $\mathrm{V}_{\mathrm{tb}}$, the CabibboKobayashi-Maskawa quark-mixing matrix (CKM), allowing non-trivial tests of the properties of this matrix in the SM [18-20]. In normal pp collision, Single Top produces mainly through two body s-channel (t-channel) Single Top in association with a b (light) quark, Wt channels and three body tbq ${ }^{\prime}$ channel. Here we focus on the study of Wt channel. This channel is invisible at the Tevatron, however, it will be important at the LHC and even comparable to Single Top s-channel production. Even though, its cross section is almost a factor 100 smaller than the most dangerous background coming from the t $\bar{t}$ process. This makes the measurement error of Wt process as large as $\sim 41 \%$ for an integrated luminosity of $10 \mathrm{fb}^{-1}$ through normal pp collision [21]. Single top production can also proceed through $\gamma \mathrm{p}$ collisions mentioned above. This time through mainly $\mathrm{Wt}$ and $\mathrm{tbq}^{\prime}$ channels. Compare these two channels, we find that cross section of Wt channel (order of $\sim 1 \mathrm{pb}$ ), is much 
larger than that of tbq' channel (order of $\sim 0.028 \mathrm{pb}$ ), and becomes the most important Single Top production channel at the $\gamma$ p collision at the LHC. This is different from normal pp collision that tbq' production channel accounts for $44.2 \%$ (39\%) of the total Single Top quark production cross section, while Wt channel stands only $28 \%(5 \%)$ at LHC (Tevatron) [22]. In contrast, Wt channel stands over $40 \%$ of the top quark photoproductions, since the top pair photoproduction has a cross section of only $\sim 1.5 \mathrm{pb}$. Enhancement of the ratio $\sigma_{\mathrm{Wt}} / \sigma_{\mathrm{t} \overline{\mathrm{t}}}$ might certainly be a good feature of related measurements through $\mathrm{Wt}$ channel.

First results on the measurement of the $\mathrm{V}_{\text {tb }}$ matrix element using $\mathrm{Wt}$ photoproduction are presented in refs. [23, 24]. There comes the conclusion that the expected error on the measurement of $\mathrm{V}_{\mathrm{tb}}$ is $16.9 \%$ for the semi-leptonic channel and $10.1 \%$ for the leptonic one after $10 \mathrm{fb}^{-1}$ of integrated luminosity, while the expected uncertainty from the equivalent study based on partonic interactions is $14 \%$ [21] using the same integrated luminosity, showing that photoproduction is at least competitive with partonic-based studies and that the combination of both studies could lead to significant improvement of the error.

In addition to the $\mathrm{V}_{\text {tb }}$ measurement, Wt photoproduction can also be used to study the $\mathrm{W}$-t-b vertex and test precisely the V-A structure of the charged current weak interaction of the top quark. Anomalous measurement of this vertex may lead direct evidence of new physics beyond the SM. It may manifest itself via either loop effects or inducing non-SM weak interactions to introduce new Single Top production channels. Typical studies involve, i.e., measuring anomalous W-t-b coupling in ep collision $[25,26]$, in normal pp collision [2735] as well as in $\gamma \mathrm{p}$ collision at the LHC [36]. Ref. [36] studies $\mathrm{pp} \rightarrow \mathrm{p} \gamma \mathrm{p} \rightarrow \mathrm{pW}^{ \pm} \mathrm{t}+\mathrm{Y}$ up to the leading order (LO) induced by anomalous W-t-b coupling. In this case, SM Wt photoproduction turns to its irreducible background. Moreover, a lot of studies are performed at the $\gamma \mathrm{p}$ colliders, i.e., testing anomalous gauge boson couplings [37-42] or probing flavour changing neutral currents (FCNC) through Single Top photoproduction [43, 44], etc. In cases like these, SM Wt photoproduction turns to be a most important reducible background that needs precise measurement and analysis. Furthermore, determining Wt production cross sections and once compared with experiments, will provide a direct access to the bottom quark parton density in nucleons and help understand the nature of the $\mathrm{b}$ quark parton distribution function (PDF).

As a result, accurate theoretical predictions including higher order QCD corrections for Wt photoproduction are needed. NLO QCD corrections for Wt production in the normal pp collisions have already been well studied [45-52]. In this paper, we present its production at the $\gamma \mathrm{p}$ collision for the first time, assuming a typical LHC multipurpose forward detector, including the NLO QCD corrections via the main reaction $\mathrm{pp} \rightarrow \mathrm{p} \gamma \mathrm{p} \rightarrow \mathrm{pW}^{ \pm} \mathrm{t}+\mathrm{Y}$. Typically, we use the Five-Flavor-Number Schemes (5FNS) with massless b quark mass assumption through the whole calculation. Our paper is organized as follow: we build the calculation framework in section 2 including brief introduction to the Equivalent Photon Approximation (EPA), general inelastic photoproduction cross section, LO and QCD NLO Wt photoproductions. Section 3 is arranged to present the input parameters, cross checks and numerical results of our study. Finally we summarize our conclusions in the last section. 


\section{Calculation framework}

\subsection{Equivalent photon approximation}

In our paper, we focus on the discussion of photoproductions $\mathrm{pp} \rightarrow \mathrm{p} \gamma \mathrm{p} \rightarrow \mathrm{pXY}$ through $\gamma \mathrm{p}$ collisioins see figure 1 [right figure]. Photoproduction is a class of processes in which one of the two interacting protons is not destroyed during the collision but survive into the final state with additional particle (or particles) state(s). Protons of this kind are named intact or forward protons. The kinematics of a forward proton is often described by means of the reduced energy loss $\xi$, which is also defined as the forward detector acceptance:

$$
\xi=\frac{\Delta \mathrm{E}}{\mathrm{E}}=\frac{\mathrm{E}-\mathrm{E}^{\prime}}{\mathrm{E}}
$$

where $\mathrm{E}$ is the initial energy of the beam and $\mathrm{s}=4 \mathrm{E}^{2}$ is the square of the centre-of-mass energy. $\mathrm{E}^{\prime}$ is the energy after the interaction and $\Delta E$ is the energy that the proton lost in the interaction. Compare to the usual pp Deep Inelastic Scattering (DIS), $\gamma \gamma$ and $\gamma \mathrm{p}$ collisions can provide more clean environment. Compare themselves, $\gamma \gamma$ collisions can be cleaner than the $\gamma \mathrm{p}$ collisions. However, $\gamma \mathrm{p}$ collisions have higher energy and effective luminosity with respect to $\gamma \gamma$ collisions.

Processes through $\gamma \gamma$ or $\gamma$ p interactions involve photon exchange with proton beams at the LHC which can be described by the appropriate framework of equivalent photon (or Weizsäcker-Williams) approximation (EPA) [53-55]. In the framework of EPA, emitted quasi-real photons from the protons have a low virtuality and scattered with small angles from the beam pipe. Therefore the emitters proton should also be scattered with a small angle and tagged by the forward detectors with some momentum fraction loss $\xi$ given in eq. (2.1). Higher $\xi$ can be obtained with the closer installation of the forward detectors from the interaction points. The emitted quasi-real photons by the emitters protons with small angles show a spectrum of virtuality $\mathrm{Q}^{2}$ and the energy $\mathrm{E}_{\gamma}$. This is described by the EPA which differs from the point-like electron (positron) case by taking care of the electromagnetic form factors in the equivalent photon spectrum and effective photon luminosity:

$$
\frac{\mathrm{dN}_{\gamma}}{\mathrm{dE}_{\gamma} \mathrm{dQ}^{2}}=\frac{\alpha}{\pi} \frac{1}{\mathrm{E}_{\gamma} \mathrm{Q}^{2}}\left[\left(1-\frac{\mathrm{E}_{\gamma}}{\mathrm{E}}\right)\left(1-\frac{\mathrm{Q}_{\mathrm{min}}^{2}}{\mathrm{Q}^{2}}\right) \mathrm{F}_{\mathrm{E}}+\frac{\mathrm{E}_{\gamma}^{2}}{2 \mathrm{E}^{2}} \mathrm{~F}_{\mathrm{M}}\right]
$$

with

$$
\begin{aligned}
& \mathrm{Q}_{\min }^{2}=\left(\frac{M_{\mathrm{inv}}^{2} E}{E-E_{\gamma}}-M_{P}^{2}\right) \frac{E_{\gamma}}{E}, \quad \mathrm{~F}_{\mathrm{E}}=\frac{4 \mathrm{M}_{\mathrm{p}}^{2} \mathrm{G}_{\mathrm{E}}^{2}+\mathrm{Q}^{2} \mathrm{G}_{\mathrm{M}}^{2}}{4 \mathrm{M}_{\mathrm{p}}^{2}+\mathrm{Q}^{2}}, \\
& \mathrm{G}_{\mathrm{E}}^{2}=\frac{\mathrm{G}_{\mathrm{M}}^{2}}{\mu_{\mathrm{p}}^{2}}=\left(1+\frac{\mathrm{Q}^{2}}{\mathrm{Q}_{0}^{2}}\right)^{-4}, \quad \mathrm{~F}_{\mathrm{M}}=\mathrm{G}_{\mathrm{M}}^{2}, \quad \mathrm{Q}_{0}^{2}=0.71 \mathrm{GeV}^{2},
\end{aligned}
$$

where $\alpha$ is the fine-structure constant, $\mathrm{E}$ is the energy of the incoming proton beam. which is related to the quasi-real photon energy by $\mathrm{E}_{\gamma}=\xi \mathrm{E} . \mathrm{M}_{\mathrm{p}}$ is the mass of the proton and $\mathrm{M}_{\mathrm{inv}}$ is the invariant mass of the final state. $\mu_{\mathrm{p}}^{2}=7.78$ is the magnetic moment of the 
proton. $F_{E}$ and $F_{M}$ are functions of the electric and magnetic form factors given in the dipole approximation.

Many phenomenological studies on photon induced processes are summarized here involve: standard model productions [56], supersymmetry [57-62], extra dimensions [6366], unparticle physics [67], top triangle moose model [68], gauge boson self-interactions [3742, 69-72], neutrino electromagnetic properties [73-75], the top quark physics [23, 24, 36, 43, 44], dark matter searches [76] and triplet Higgs production [77], etc.

\subsection{General $\gamma$ p photoproduction cross section}

We denote the general photoproduction processes at the LHC, no metter at LO or NLO level, as

$$
\mathrm{pp} \rightarrow \mathrm{p} \gamma \mathrm{p} \rightarrow \mathrm{p}+\gamma+\mathrm{q} / \overline{\mathrm{q}} / \mathrm{g} \rightarrow \mathrm{p}+\underbrace{i+j+k+\ldots}_{\mathrm{X}}+\mathrm{Y}
$$

with $\mathrm{q}=\mathrm{u}, \mathrm{d}, \mathrm{c}, \mathrm{s}, \mathrm{b}$ and $\mathrm{i}, \mathrm{j}, \mathrm{k}, \ldots$ the final state particles. The hadronic cross section at the LHC can be converted by integrating $\gamma+\mathrm{q} / \overline{\mathrm{q}} / \mathrm{g} \rightarrow \mathrm{i}+\mathrm{j}+\mathrm{k}+\ldots$ over the photon $\left(\mathrm{dN}\left(\mathrm{x}, \mathrm{Q}^{2}\right)\right)$, gluon and quark $\left(\mathrm{G}_{\mathrm{g}, \mathrm{q} / \mathrm{p}}\left(\mathrm{x}_{2}, \mu_{\mathrm{f}}\right)\right)$ spectra:

$$
\begin{array}{r}
\sigma_{\gamma \mathrm{p}}=\sum_{\mathrm{j}=\mathrm{q}, \overline{\mathrm{q}}, \mathrm{g}} \int_{\frac{\mathrm{M}_{\mathrm{inv}}}{\sqrt{\mathrm{s}}}}^{\sqrt{\xi_{\max }}} 2 \mathrm{zdz} \int_{\operatorname{Max}\left(\mathrm{z}^{2}, \xi_{\min }\right)}^{\xi_{\max }} \frac{\mathrm{dx}_{1}}{\mathrm{x}_{1}} \int_{\mathrm{Q}_{\min }^{2}}^{\mathrm{Q}_{\max }^{2}} \frac{\mathrm{dN}_{\gamma}\left(\mathrm{x}_{1}\right)}{\mathrm{dE}_{\gamma} \mathrm{dQ}^{2}} \mathrm{G}_{\mathrm{g}, \mathrm{q} / \mathrm{p}}\left(\frac{\mathrm{z}^{2}}{\mathrm{x}_{1}}, \mu_{\mathrm{f}}\right) \\
\cdot \int \frac{1}{\operatorname{avgfac}} \frac{\left|\mathcal{M}_{\mathrm{n}}\left(\gamma \mathrm{j} \rightarrow \mathrm{klm} \ldots, \hat{\mathrm{s}}=\mathrm{z}^{2} \mathrm{~s}\right)\right|^{2}}{2 \hat{\mathrm{s}}(2 \pi)^{3 \mathrm{n}-4}} \mathrm{~d} \Phi_{\mathrm{n}}
\end{array}
$$

where $\mathrm{x}_{1}$ is the ratio between scattered quasi-real photons and incoming proton energy $\mathrm{x}_{1}=\mathrm{E}_{\gamma} /$ E. $\xi_{\min }\left(\xi_{\max }\right)$ are its lower (upper) limits which means that the forward detector acceptance satisfies $\xi_{\min } \leq \xi \leq \xi_{\max } . \mathrm{x}_{2}$ is the momentum fraction of the proton momentum carried by the gluon (quark). The quantity $\hat{\mathrm{s}}=\mathrm{z}^{2} \mathrm{~s}$ is the effective c.m.s. energy with $\mathrm{z}^{2}=\mathrm{x}_{1} \mathrm{x}_{2} . \mathrm{s}=4 \mathrm{E}^{2}$ mentioned above and $\mathrm{M}_{\mathrm{inv}}$ is the total mass of the related final states. $2 \mathrm{z} / \mathrm{x}_{1}$ is the Jacobian determinant when transform the differentials from $\mathrm{dx}_{1} \mathrm{dx}_{2}$ into $\mathrm{dx}_{1} \mathrm{dz}$. $\mathrm{G}_{\mathrm{g}, \mathrm{q} / \mathrm{p}}\left(\mathrm{x}, \mu_{\mathrm{f}}\right)$ represent the gluon (quark) parton density functions, $\mu_{\mathrm{f}}$ is the factorization scale. $\mathrm{f}=\frac{\mathrm{dN}}{\mathrm{dE}_{\gamma} \mathrm{dQ}^{2}}$ is the $\mathrm{Q}^{2}$ dependent relative luminosity spectrum present in eq. (2.2). $\mathrm{Q}_{\max }^{2}=2 \mathrm{GeV}^{2}$ is the maximum virtuality. $\frac{1}{\text { avgfac }}$ is the product of the spin-average factor, the color-average factor and the identical particle factor. $\left|\mathcal{M}_{\mathrm{n}}\right|^{2}$ presents the squared nparticle matrix element and divided by the flux factor $\left[2 \hat{\mathrm{s}}(2 \pi)^{3 \mathrm{n}-4}\right]$. The $\mathrm{n}$-body phase space differential $\mathrm{d} \Phi_{\mathrm{n}}$ and its integral $\Phi_{\mathrm{n}}$ depend only on $\hat{\mathrm{s}}$ and particle masses $\mathrm{m}_{\mathrm{i}}$ due to Lorentz invariance:

$$
\begin{aligned}
\Phi_{\mathrm{n}}\left(\hat{\mathrm{s}}, \mathrm{m}_{1}, \mathrm{~m}_{2}, \ldots, \mathrm{m}_{\mathrm{n}}\right) & =\int \mathrm{d} \Phi_{\mathrm{n}}\left(\hat{\mathrm{s}}, \mathrm{m}_{1}, \mathrm{~m}_{2}, \ldots, \mathrm{m}_{\mathrm{n}}\right) \\
& =\int \delta^{4}\left(\left(\mathrm{p}_{\mathrm{i}}+\mathrm{p}_{\mathrm{j}}\right)-\sum_{\mathrm{k}=1}^{\mathrm{n}} \mathrm{p}_{\mathrm{k}}\right) \prod_{\mathrm{k}=1}^{\mathrm{n}} \mathrm{d}^{4} \mathrm{p}_{\mathrm{k}} \delta\left(\mathrm{p}_{\mathrm{k}}^{2}-\mathrm{m}_{\mathrm{k}}^{2}\right) \Theta\left(\mathrm{p}_{\mathrm{k}}^{0}\right)
\end{aligned}
$$

with $\mathrm{i}$ and $\mathrm{j}$ denoting the incident particles and $\mathrm{k}$ running over all outgoing particles. 


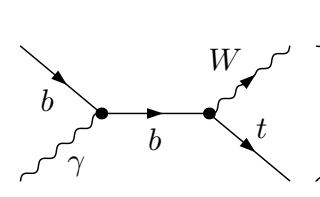

(1)

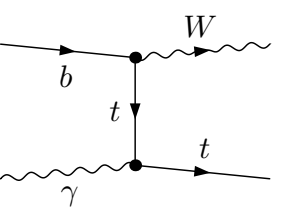

$(2)$

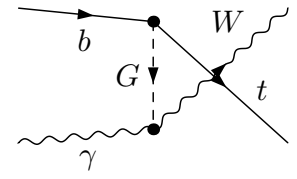

(3)

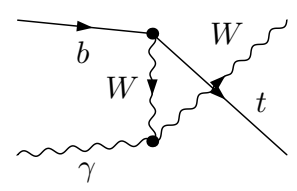

(4)

Figure 2. Tree level Feynman diagrams for $\gamma \mathrm{b} \rightarrow \mathrm{W}^{-} \mathrm{t}$ in the SM.

\subsection{Wt photoproduction at leading order}

We denote the Wt photoproduction process as:

$$
\mathrm{pp} \rightarrow \mathrm{p} \gamma \mathrm{p} \rightarrow \mathrm{p} \gamma\left(\mathrm{p}_{1}\right) \mathrm{b}\left(\mathrm{p}_{2}\right) \rightarrow \mathrm{pW}^{-}\left(\mathrm{p}_{3}\right) \mathrm{t}\left(\mathrm{p}_{4}\right)+\mathrm{Y}
$$

where $p_{i}$ are the particle four momentums. There are four LO Feynman diagrams for this partonic process as shown in figure 2. There figure 2(1) and figures 2(2)-2(4) are the schannel and t-channel diagrams for the partonic process, respectively. Figure 2(3) include b-t-G vertex that can be safely omitted in the massless b quark assumption. We only consider the $\mathrm{W}^{-}$t production while its charge-conjugate contribution is the same [50]. In order to describe the process $\gamma\left(\mathrm{p}_{1}\right) \mathrm{b}\left(\mathrm{p}_{2}\right) \rightarrow \mathrm{W}^{-}\left(\mathrm{p}_{3}\right) \mathrm{t}\left(\mathrm{p}_{4}\right)$ we choose the c.m.s. $\left(\mathbf{p}_{1}+\mathbf{p}_{2}=0\right)$ with the momentum directed along z-axis. In c.m.s. the particle momentum read

$$
\begin{array}{ll}
\mathrm{p}_{1}=\frac{\sqrt{\hat{\mathrm{s}}}}{2}\left(\mathrm{e}_{1}, 0,0, \mathrm{e}_{1 \mathrm{z}}\right), & \mathrm{p}_{3}=\frac{\sqrt{\hat{\mathrm{s}}}}{2}\left(\mathrm{e}_{3}, \mathrm{e}_{3 \mathrm{x}}, \mathrm{e}_{3 \mathrm{y}}, \mathrm{e}_{3 \mathrm{z}}\right) \\
\mathrm{p}_{2}=\frac{\sqrt{\hat{\mathrm{s}}}}{2}\left(\mathrm{e}_{2}, 0,0, \mathrm{e}_{2 \mathrm{z}}\right), & \mathrm{p}_{4}=\frac{\sqrt{\hat{\mathrm{s}}}}{2}\left(\mathrm{e}_{4}, \mathrm{e}_{4 \mathrm{x}}, \mathrm{e}_{4 \mathrm{y}}, \mathrm{e}_{4 \mathrm{z}}\right) .
\end{array}
$$

Here notation of $\mathrm{e}_{\mathrm{i} / \mathrm{ix}}$ equal $\mathrm{p}_{\mathrm{i} / \mathrm{ix}} /(\sqrt{\hat{\mathrm{s}}} / 2)$ and is needed in our following description.

The LO cross section for the partonic process $\gamma \mathrm{b} \rightarrow \mathrm{W}^{-} \mathrm{t}$ is obtained by using the following formula

$$
\hat{\sigma}^{\mathrm{LO}}\left(\hat{\mathrm{s}}, \gamma \mathrm{b} \rightarrow \mathrm{W}^{-} \mathrm{t}\right)=\frac{(2 \pi)^{4}}{4\left|\mathbf{p}_{1}\right| \sqrt{\hat{\mathrm{s}}}} \int \bar{\sum}\left|\mathcal{M}^{\mathrm{LO}}\right|^{2} \mathrm{~d} \Phi_{2}
$$

where $\mathrm{d} \Phi_{2}$ is the two-body phase-space element, and $\mathbf{p}_{1}$ is the momentum of the initial photon in the c.m.s.. The integration is performed over the two-body phase space of the final particles $\mathrm{W}^{-} \mathrm{t}$. The summation is taken over the spins and colors of the initial and final states, and the bar over the summation indicates averaging over the intrinsic degrees of freedom of initial partons.

The LO total cross section for $\mathrm{pp} \rightarrow \mathrm{p} \gamma \mathrm{p} \rightarrow \mathrm{pW}^{-} \mathrm{t}$ can be expressed as

$$
\begin{aligned}
& \sigma^{\mathrm{LO}}\left(\mathrm{pp} \rightarrow \mathrm{p} \gamma \mathrm{p} \rightarrow \mathrm{pW}^{-} \mathrm{t}+\mathrm{Y}\right) \\
& =\int_{\frac{\mathrm{M}_{\mathrm{inv}}}{\sqrt{\mathrm{s}}}}^{\sqrt{\xi_{\max }}} 2 \mathrm{zdz} \int_{\operatorname{Max}\left(\mathrm{z}^{2}, \xi_{\min }\right)}^{\xi_{\max }} \frac{\mathrm{dx}_{1}}{\mathrm{x}_{1}} \mathrm{f}_{\gamma / \mathrm{P}_{\mathrm{A}}}\left(\mathrm{x}_{1}\right) \mathrm{G}_{\mathrm{b} / \mathrm{P}_{\mathrm{B}}}\left(\frac{\mathrm{z}^{2}}{\mathrm{x}_{1}}, \mu_{\mathrm{f}}\right) \hat{\sigma}^{\mathrm{LO}}\left(\gamma \mathrm{b} \rightarrow \mathrm{W}^{-} \mathrm{t}, \mathrm{z}^{2} \mathrm{~s}, \mu_{\mathrm{f}}, \mu_{\mathrm{r}}\right) \\
& \quad+(\mathrm{A} \leftrightarrow \mathrm{B}) .
\end{aligned}
$$

There $\mathrm{G}_{\mathrm{i} / \mathrm{P}_{\mathrm{j}}}, \mathrm{i}=\mathrm{b}, \mathrm{j}=\mathrm{A}, \mathrm{B}$ represent the PDFs of parton $\mathrm{i}$ in proton $\mathrm{P}_{\mathrm{j}}, \mu_{\mathrm{f}}$ and $\mu_{\mathrm{r}}$ are the factorization and renormalization scales separately. Here we use $\mathrm{f}_{\gamma / \mathrm{P}_{\mathrm{A}}}\left(\mathrm{x}_{1}\right)$ to take place of 
the $\int_{Q_{\min }^{2}}^{\mathrm{Q}_{\max }^{2}} \frac{\mathrm{dN}_{\gamma}\left(\mathrm{x}_{1}\right)}{\mathrm{dE}_{\gamma} \mathrm{dQ}^{2}}$ in eq. (2.5) for simplicity. And we address here that during calculation, we use the $\xi, \mathrm{Q}^{2}$ dependent form of eq. (2.5).

\subsection{Wt photoproduction at QCD next-to-leading order}

\subsubsection{General description}

We use the Five-Flavor-Number Scheme (5FNS) in our whole LO and QCD NLO calculations. As we see, at tree level in the 5 FNS scheme the Wt photoproduction process consists of only one partonic subprocess, namely $\gamma \mathrm{b} \rightarrow \mathrm{W}^{-} \mathrm{t}$, as illustrated in figure 2 . Indeed, Wt photoproduction can also be produced in the Four-Flavor-Number Scheme (4FNS) where the $\mathrm{b}$ quark is treated as massive and there is no b quark parton density is assumed in the initial state. In this scheme, the LO contribution starts from $\gamma \mathrm{g} \rightarrow \mathrm{W}^{-} \mathrm{t} \overline{\mathrm{b}}$ with $1 \mathrm{~b}$ tagged in the final state. The first order of QCD corrections in 4FNS consist of virtual one-loop corrections to the tree-level subprocesses as well as real corrections in the form of other two subprocesses with an additional radiated parton, namely $\gamma \mathrm{g} \rightarrow \mathrm{W}^{-} \mathrm{tb}+\mathrm{g}$ and $\gamma \mathrm{q} \rightarrow \mathrm{W}^{-} \mathrm{t} \overline{\mathrm{b}}+\overline{\mathrm{q}}$. In the $4 \mathrm{FNS}$ scheme, b quark do not enter in the computation of the running of $\alpha_{\mathrm{s}}$ and the evolution of the PDFs. Finite $\mathrm{m}_{\mathrm{b}}$ effects enter via power corrections of the type $\left(\mathrm{m}_{\mathrm{b}}^{2} / \mathrm{Q}^{2}\right)^{\mathrm{n}}$ and logarithms of the type $\log ^{\mathrm{n}}\left(\mathrm{m}_{\mathrm{b}}^{2} / \mathrm{Q}^{2}\right)$ where $\mathrm{Q}$ stands for the hard scale of the process. At the LHC, typically $\left(\mathrm{m}_{\mathrm{b}} / \mathrm{Q}\right) \ll 1$ and power corrections are suppressed, while logarithms, both of initial and final state nature, could be large. These large logarithms could in principle spoil the convergence of fixed order calculations and a resummation could be required. Up to NLO accuracy those potentially large logarithms, $\log \left(\mathrm{m}_{\mathrm{b}} / \mathrm{Q}\right)$, are replaced by $\log \left(\mathrm{p}_{\mathrm{T}, \mathrm{b}}^{\min } / \mathrm{Q}\right)$ with $\mathrm{m}_{\mathrm{b}} \ll \mathrm{p}_{\mathrm{T}, \mathrm{b}}^{\min } \leq \mathrm{Q}$ and are less significant numerically. As can be see, the difference between adopting the 5FNS and 4FNS is the ordering of the perturbative series for the production cross section. In the $4 \mathrm{FNS}$ the perturbative series is ordered strictly by powers of the strong coupling $\alpha_{\mathrm{s}}$, while in the 5FNS the introduction of the $\mathrm{b}$ quark PDF allows to resum terms of the form $\alpha_{\mathrm{s}}^{\mathrm{n}} \log \left(\mu^{2} / \mathrm{m}_{\mathrm{b}}^{2}\right)^{\mathrm{m}}$ at all orders in $\alpha_{\mathrm{s}}$. If all orders in perturbation theory were taken into account, these two schemes are identical in describing logarithmic effects. But the way of ordering in the perturbative expansion is different and at any finite order the results might be different. Many works have been done in the comparison of the $5 \mathrm{FNS}$ and $4 \mathrm{FNS}$ Schemes, see refs. [78-84], etc. A latest comparison in the $4 \mathrm{FNS}$ and $5 \mathrm{FNS}$ schemes in ref. [85] present that being often the effects of resummation very mild, 4 FNS calculations can be put to use, on the other hand, for 5NFS schemes, i.e., can typically provide quite accurate predictions for total rates and being simpler, in some cases allow the calculations to be performed at NNLO. We address the interesting of considering both schemes while here we use 5FNS in our calculation. Even in 5FNS, it will be interesting to consider two schemes [80, 81, 86-90]: one is the massless b quark scheme where we drop the mass of $b$ quark during calculation while the other is the massive $\mathrm{b}$ quark scheme where we retain it everywhere.

In our paper, we adopt the 5FNS scheme with the massless b quark assumption. In this case, the first order of $\mathrm{QCD}$ corrections to the $\mathrm{pp} \rightarrow \mathrm{p} \gamma \mathrm{p} \rightarrow \mathrm{pW}^{-} \mathrm{t}+\mathrm{Y}$ consist of:

- The QCD one-loop virtual corrections to the partonic process $\gamma \mathrm{b} \rightarrow \mathrm{W}^{-} \mathrm{t}$.

- The contribution of the real gluon radiation partonic process $\gamma \mathrm{b} \rightarrow \mathrm{W}^{-} \mathrm{t}+\mathrm{g}$. 
- The contribution of the real b-quark emission partonic process $\gamma g \rightarrow \mathrm{W}^{-} \mathrm{t}+\overline{\mathrm{b}}$.

- The corresponding contributions of the PDF counterterms.

We use the dimensional regularization method in $\mathrm{D}=4-2 \epsilon$ dimensions to isolate the ultraviolet (UV) and infrared (IR) singularities. In massless b quark scheme, we split each collinear counter-term of the $\mathrm{PDF}, \delta \mathrm{G}_{\mathrm{b} / \mathrm{P}}\left(\mathrm{x}, \mu_{\mathrm{f}}\right)(\mathrm{P}=$ proton), into two parts: the collinear gluon emission part $\delta \mathrm{G}_{\mathrm{b} / \mathrm{P}}^{\text {gluon }}\left(\mathrm{x}, \mu_{\mathrm{f}}\right)$ and the collinear b quark emission part $\delta \mathrm{G}_{\mathrm{b} / \mathrm{P}}^{\text {quark }}\left(\mathrm{x}, \mu_{\mathrm{f}}\right)$. The analytical expressions are presented as follows

$$
\delta \mathrm{G}_{\mathrm{b} / \mathrm{P}}\left(\mathrm{x}, \mu_{\mathrm{f}}\right)=\delta \mathrm{G}_{\mathrm{b} / \mathrm{P}}^{\text {gluon }}\left(\mathrm{x}, \mu_{\mathrm{f}}\right)+\delta \mathrm{G}_{\mathrm{b} / \mathrm{P}}^{\text {quark }}\left(\mathrm{x}, \mu_{\mathrm{f}}\right)
$$

with

$$
\begin{aligned}
\delta \mathrm{G}_{\mathrm{b} / \mathrm{P}}^{\text {gluon }} & =\frac{1}{\epsilon}\left[\frac{\alpha_{\mathrm{s}}}{2 \pi} \frac{\Gamma(1-\epsilon)}{\Gamma(1-2 \epsilon)}\left(\frac{4 \pi \mu_{\mathrm{r}}^{2}}{\mu_{\mathrm{f}}^{2}}\right)^{\epsilon}\right] \int_{\mathrm{x}}^{1} \frac{\mathrm{dz}}{\mathrm{z}} \mathrm{P}_{\mathrm{bb}}(\mathrm{z}) \mathrm{G}_{\mathrm{b} / \mathrm{P}}\left(\frac{\mathrm{x}}{\mathrm{z}}, \mu_{\mathrm{f}}\right) \\
\delta \mathrm{G}_{\mathrm{b} / \mathrm{P}}^{\text {quark }} & =\frac{1}{\epsilon}\left[\frac{\alpha_{\mathrm{s}}}{2 \pi} \frac{\Gamma(1-\epsilon)}{\Gamma(1-2 \epsilon)}\left(\frac{4 \pi \mu_{\mathrm{r}}^{2}}{\mu_{\mathrm{f}}^{2}}\right)^{\epsilon}\right] \int_{\mathrm{x}}^{1} \frac{\mathrm{dz}}{\mathrm{z}} \mathrm{P}_{\mathrm{bg}}(\mathrm{z}) \mathrm{G}_{\mathrm{g} / \mathrm{P}}\left(\frac{\mathrm{x}}{\mathrm{z}}, \mu_{\mathrm{f}}\right)
\end{aligned}
$$

and the explicit expressions for the splitting functions $\mathrm{P}_{\mathrm{ij}}(\mathrm{z}),(\mathrm{ij}=\mathrm{bb}, \mathrm{bg})$ can be found in ref. [91].

\subsubsection{Virtual}

The amplitude at the QCD one-loop level for the partonic process $\gamma \mathrm{b} \rightarrow \mathrm{W}^{-} \mathrm{t}$ in the SM contains the contributions of the self-energy, vertex, box and counter-term graphs which are shown in figures 3(1)-3(18). Same as the leading level that diagrams include b-t-G vertex that can be safely omitted in the massless b quark scheme. Even in the massive $b$ quark scheme, their contributions are also quite small.

To remove the UV divergences, we need to renormalize the mass of the quarks and the wave function of the quark fields. In the massless b quark assumption we introduce the following renormalization constants:

$$
\begin{aligned}
\psi_{\mathrm{b}(\mathrm{t})}^{0, \mathrm{~L}, \mathrm{R}} & =\left(1+\delta \mathrm{Z}_{\psi_{\mathrm{b}(\mathrm{t})}^{\mathrm{L}, \mathrm{R}}}\right)^{\frac{1}{2}} \psi_{\mathrm{b}(\mathrm{t})}^{\mathrm{L}, \mathrm{R}}, \\
\mathrm{m}_{\mathrm{t}}^{0} & =\mathrm{m}_{\mathrm{t}}+\delta \mathrm{m}_{\mathrm{t}},
\end{aligned}
$$

where $\mathrm{m}_{\mathrm{t}}$ are the top-quark mass. $\psi_{\mathrm{b}(\mathrm{t})}^{\mathrm{L}, \mathrm{R}}$ denote the fields of bottom (top) quark. For the masses and wave functions of the fields are renormalized in the the on-shell scheme and the relevant counter-terms are expressed as

$$
\begin{aligned}
\delta \mathrm{Z}_{\psi_{\mathrm{t}}}^{\mathrm{L}, \mathrm{R}} & =-\frac{\alpha_{\mathrm{s}}}{4 \pi} \mathrm{C}_{\mathrm{F}}\left[\Delta_{\mathrm{UV}}+2 \Delta_{\mathrm{IR}}+4+3 \ln \left(\frac{\mu_{\mathrm{r}}^{2}}{\mathrm{~m}_{\mathrm{t}}^{2}}\right)\right], \\
\frac{\delta \mathrm{m}_{\mathrm{t}}}{\mathrm{m}_{\mathrm{t}}} & =-\frac{\alpha_{\mathrm{s}}}{4 \pi} \mathrm{C}_{\mathrm{F}}\left[3 \Delta_{\mathrm{UV}}+4+3 \ln \left(\frac{\mu_{\mathrm{r}}^{2}}{\mathrm{~m}_{\mathrm{t}}^{2}}\right)\right],
\end{aligned}
$$




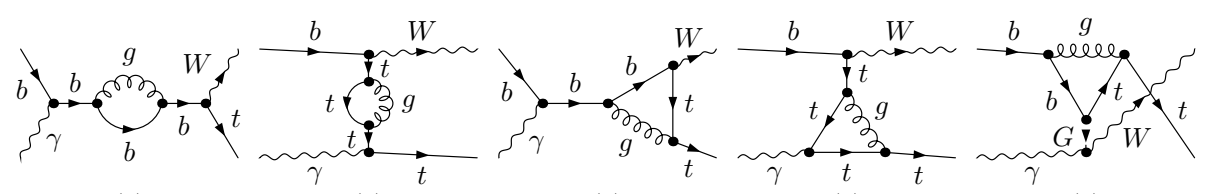

(1)

(2)

(3)

(4)

(5)

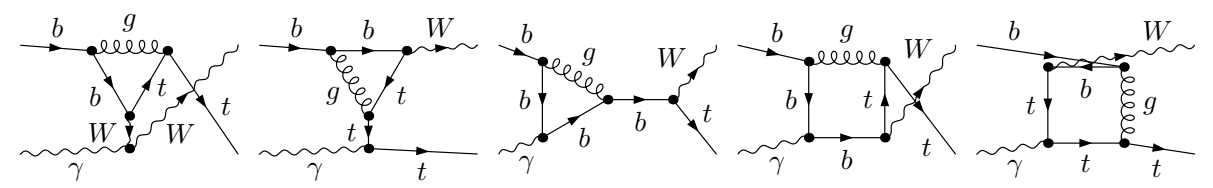

(6)

(7)

(8)

(9)

(10)

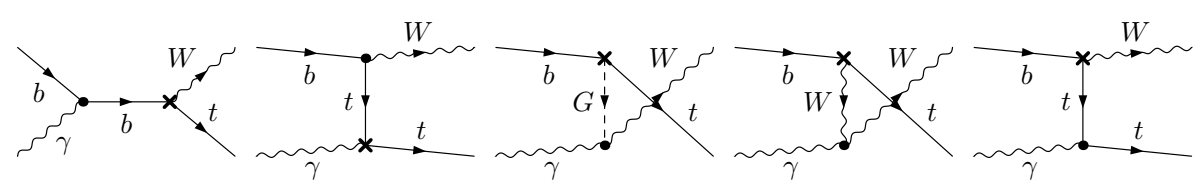

(11)

(12)

(13)

(14)

(15)

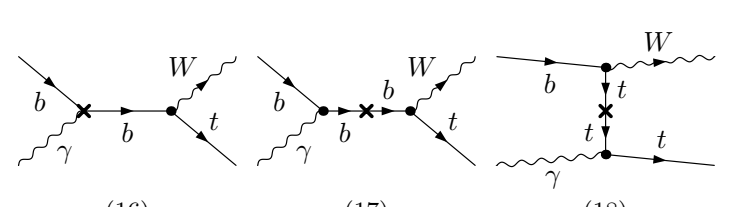

(16)

(17)

(18)

Figure 3. QCD one loop Feynman diagrams for $\gamma \mathrm{b} \rightarrow \mathrm{W}^{-} \mathrm{t}$ in the SM.

with $\mathrm{C}_{\mathrm{F}}=\frac{4}{3}, \Delta_{\mathrm{UV}(\mathrm{IR})}=\frac{1}{\epsilon_{\mathrm{UV}(\mathrm{IR})}} \Gamma\left(1+\epsilon_{\mathrm{UV}(\mathrm{IR})}\right)(4 \pi)^{\epsilon_{\mathrm{UV}(\mathrm{IR})}}$ refer to the $\mathrm{UV}(\mathrm{IR})$ divergences. For massless $b$ quark, there is no need to renormalize the mass of bottom and we use modified minimal subtraction $(\overline{\mathrm{MS}})$ scheme for $\mathrm{b}$ field as:

$$
\delta \mathrm{Z}_{\psi_{\mathrm{b}}}^{\mathrm{L}, \mathrm{R}}=-\frac{\alpha_{\mathrm{S}}}{4 \pi} \mathrm{C}_{\mathrm{F}}\left[\Delta_{\mathrm{UV}}-\Delta_{\mathrm{IR}}\right]
$$

UV singularities are regulated by adding renormalization part to the virtual corrections only leaving IR singularities that will be removed by combining the real emission corrections. We calculate the virtual one-loop corrections $\left(\sigma^{\mathrm{V}}\right)$ using a Feynman diagram approach based on FeynArts, FormCalc and our modified LoopTools (FFL) [92-95] packages. Tensor one loop integrals are checked with OneLoop [96] and QCDLoop [97] packages.

\subsubsection{Parton radiation}

The first order of QCD corrections also consist of the real corrections in the form of other two subprocesses with an additional radiated parton, namely gluon emission and real (anti) quark emission presented as

$$
\begin{aligned}
& \gamma\left(\mathrm{p}_{1}\right) \mathrm{b}\left(\mathrm{p}_{2}\right) \rightarrow \mathrm{W}^{-}\left(\mathrm{p}_{3}\right) \mathrm{t}\left(\mathrm{p}_{4}\right) \mathrm{g}\left(\mathrm{p}_{5}\right) \\
& \gamma\left(\mathrm{p}_{1}\right) \mathrm{g}\left(\mathrm{p}_{2}\right) \rightarrow \mathrm{W}^{-}\left(\mathrm{p}_{3}\right) \mathrm{t}\left(\mathrm{p}_{4}\right) \overline{\mathrm{b}}\left(\mathrm{p}_{5}\right)
\end{aligned}
$$

with Feynman diagrams depicted in figure 4 and figure 5, respectively.

In the massless b quark scheme, singularities associated with initial state collinear gluon emission are absorbed into the definition of the PDFs, see in eq. (2.12). We employ 


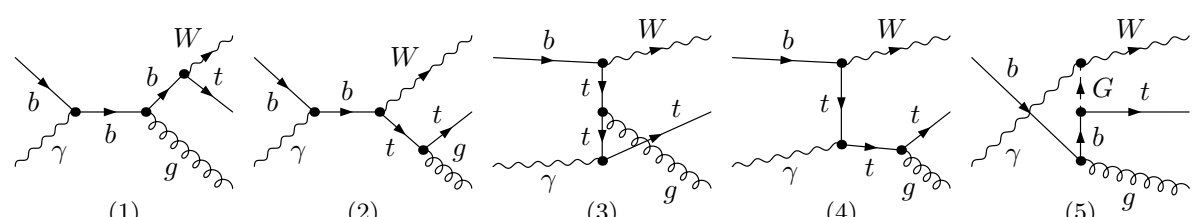

(1)

(2)

(3)

(4)

(5)

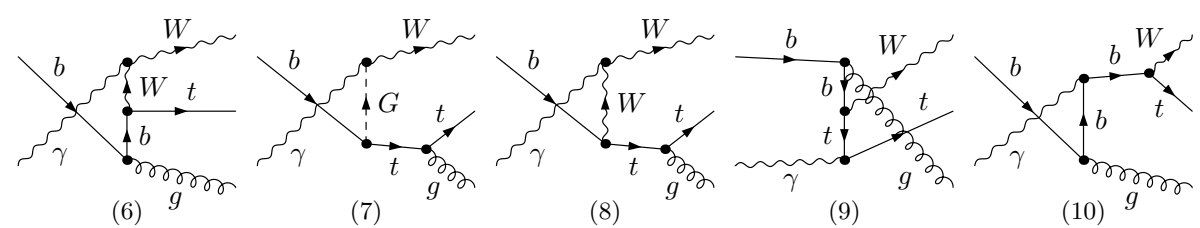

Figure 4. The tree parton level Feynman diagrams for the real gluon emission subprocess $\gamma \mathrm{b} \rightarrow$ $\mathrm{W}^{-}$tg related to eq. (2.16).
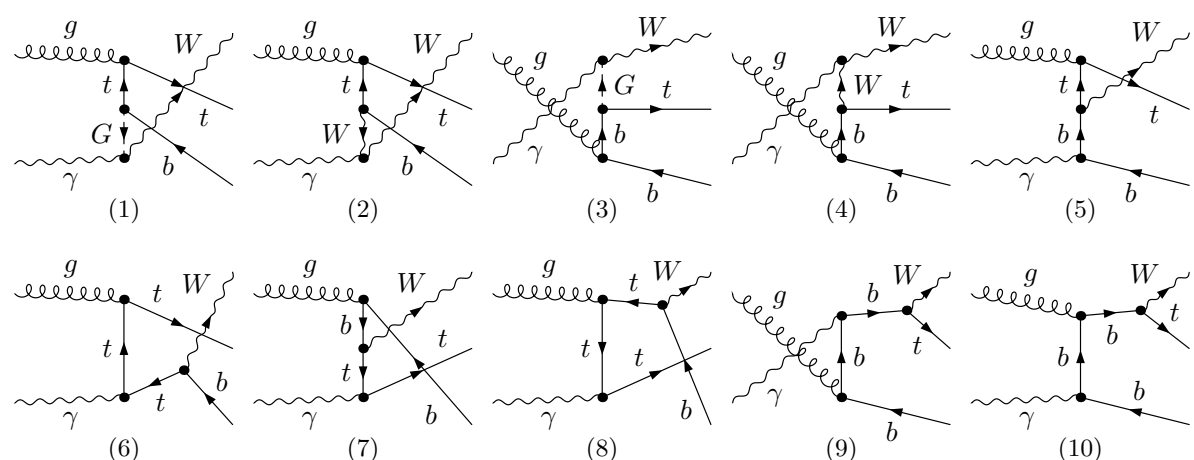

Figure 5. The tree parton level Feynman diagrams for the real light-(anti)quark emission subprocess $\gamma \mathrm{g} \rightarrow \mathrm{W}^{-} \mathrm{t} \overline{\mathrm{b}}$ related to eq. (2.17).

the $\overline{\mathrm{MS}}$ scheme for the parton distribution functions. Similar to the virtual part, we utilize dimensional regularization (DR) to control the singularities of the radiative corrections, which are organized using the two cutoff phase space slicing (TCPSS) method [91]. Since we treat the $\mathrm{b}$ quark as massless, there is collinear IR singularity which is regularized by $1 / \epsilon$ in the DR scheme. This term is canceled by the corresponding contribution in the b quark PDF counterterm, in other words, absorbed by the b quark PDF. This cancellation has been checked both analytically and numerically, therefore, avoid double counting problem. We adopt TCPSS to isolate the IR singularities by introducing two cutoff parameters $\delta_{\mathrm{s}}$ and $\delta_{\mathrm{c}}$. An arbitrary small $\delta_{\mathrm{s}}$ separates the three-body final state phase space into two regions: the soft region $\left(\mathrm{E}_{5} \leq \delta_{\mathrm{s}} \sqrt{\hat{\mathrm{s}}} / 2\right)$ and the hard region $\left(\mathrm{E}_{5}>\delta_{\mathrm{s}} \sqrt{\hat{\mathrm{s}}} / 2\right)$. The $\delta_{\mathrm{c}}$ separates hard region into the hard collinear $(\mathrm{HC})$ region and hard noncollinear $(\overline{\mathrm{HC}})$ region. The criterion for separating the HC region is described as follows: the region for real gluon/light quark emission with $\hat{\mathrm{s}}_{15}\left(\right.$ or $\left.\hat{\mathrm{s}}_{25}\right)<\delta_{\mathrm{c}} \hat{\mathrm{s}}\left(\right.$ where $\left.\hat{\mathrm{s}}_{\mathrm{ij}}=\left(\mathrm{p}_{\mathrm{i}}+\mathrm{p}_{\mathrm{j}}\right)^{2}\right)$ is called the HC region. Otherwise it is called the $\overline{\mathrm{HC}}$ region.

At QCD NLO, some of the contributions representing the emission of an additional parton require special attention. For example, when we calculate the remain part in real radiation corrections $\gamma \mathrm{g} \rightarrow \mathrm{Wtb}$ (eq. (2.17)), appropriate crossing of the diagrams shown in figure 5 should be included. Some of the diagrams which produce a final state consisting 
of a W, an on-shell top quark and a b quark are particularly problematic. During phase space integration, one need to integrate over the region $\mathrm{M}_{\mathrm{Wb}}^{2}=\left(\mathrm{p}_{\mathrm{W}}+\mathrm{p}_{\mathrm{b}}\right)$. If in this case, a resonant $\mathrm{t}$ propagator (with flowing momentum equal or close to $\mathrm{p}_{\mathrm{W}}+\mathrm{p}_{\mathrm{b}}$ ) is encountered, a divergence will arise. Actually these diagrams can be interpreted as the production of a $t \overline{\mathrm{t}}$ pair at $\mathrm{LO}$, with subsequent decay of the top into a $\mathrm{Wb}$ system. This is the well known interference between $\mathrm{Wt}$ and $t \overline{\mathrm{t}}$ production, namely doubly resonant. Such resonant becomes extremely large in certain phase space region and renders the perturbative computation of the Wt cross section meaningless, thus should be preferable excluded from the NLO corrections to the Wt process.

Several approaches have been outlined in the literature, i.e., making a cut on the invariant mass of the $\mathrm{Wb}$ system to prevent the $\mathrm{t}$ propagator from becoming resonant [22], subtracting the contribution from the resonant diagrams so that no on-shell piece remains [18, 98], bottom quark PDF method, technically, perform calculation of the Wt process by applying a veto on the $\mathrm{p}_{\mathrm{T}}$ of the additional $\mathrm{b}$ quark that appears at next-toleading order aids the separation of this process from doubly-resonant $t \bar{t}$ production [51], etc $[50,99]$. Here in our case we use the PROSPINO scheme $[100,101]$ which is defined as a replacement of the Breit-Wigner propagator

$$
\begin{aligned}
\frac{|\mathcal{M}|^{2}\left(\mathrm{~s}_{\mathrm{Wb}}\right)}{\left(\mathrm{s}_{\mathrm{Wb}}-\mathrm{m}_{\mathrm{t}}^{2}\right)^{2}+\mathrm{m}_{\mathrm{t}}^{2} \Gamma_{\mathrm{t}}^{2}} \rightarrow & \frac{|\mathcal{M}|^{2}\left(\mathrm{~s}_{\mathrm{Wb}}\right)}{\left(\mathrm{s}_{\mathrm{Wb}}-\mathrm{m}_{\mathrm{t}}^{2}\right)^{2}+\mathrm{m}_{\mathrm{t}}^{2} \Gamma_{\mathrm{t}}^{2}} \\
& -\frac{|\mathcal{M}|^{2}\left(\mathrm{~m}_{\mathrm{t}}^{2}\right)}{\left(\mathrm{s}_{\mathrm{Wb}}-\mathrm{m}_{\mathrm{t}}^{2}\right)^{2}+\mathrm{m}_{\mathrm{t}}^{2} \Gamma_{\mathrm{t}}^{2}} \Theta\left(\hat{\mathrm{s}}-4 \mathrm{~m}_{\mathrm{t}}^{2}\right) \Theta\left(\mathrm{m}_{\mathrm{t}}-\mathrm{m}_{\mathrm{W}}\right) .
\end{aligned}
$$

This subtraction scheme helps to avoid double counting and to not artificially ruin the convergence of the perturbative QCD description of these production channels with the remove of on-shell particle contributions from the associated production. This scheme has be done in some other refs like [102-105], etc.

Some attention should be paid to the light-(anti)quark emission subprocess, see figures 5(5) and 5(10). Contributions from these two diagrams can be considered as part of the NLO EW corrections to the LO process pp $\rightarrow \mathrm{gb} \rightarrow$ Wt through normal pp collision. Since we concentrate on the photoproduction of $\mathrm{pp} \rightarrow \mathrm{p} \gamma \mathrm{p} \rightarrow \mathrm{pW}^{-} \mathrm{t}$ where forward protons are considered, the pp $\rightarrow \mathrm{gb} \rightarrow \mathrm{Wt}$ and its full NLO EW corrections are not taken into account. Then, the subprocesses in figures 5(5) and 5(10) are defined applying a small $\mathrm{p}_{\mathrm{T}}$ cut on the tagged $\mathrm{b}$ quark, which regularize the collinear splitting of the photon into $a \mathrm{~b} \bar{b}$ pair. Indeed, the choice of specific kinematical cuts to select events in the forward region (small $\mathrm{p}_{\mathrm{T}}$ cut applied on the tagged $\mathrm{b}$ quark) forces the contributions from these two diagrams to be quite small. Thus, even when one is forced to consider them as part of the NLO QCD corrections to the $\mathrm{pp} \rightarrow \mathrm{p} \gamma \mathrm{p} \rightarrow \mathrm{pWt}$ production process, their contribution results for only but a tiny theoretical uncertainty.

Then the cross section for each of the real emission partonic processes can be written as $\hat{\sigma}^{\mathrm{R}}=\hat{\sigma}^{\mathrm{S}}+\hat{\sigma}^{\mathrm{H}}=\hat{\sigma}^{\mathrm{S}}+\hat{\sigma}^{\mathrm{HC}}+\hat{\sigma}^{\mathrm{HC}}$. After integrating over the photon and quark spectra, we get the real contributions as $\sigma^{\mathrm{R}}=\sigma^{\mathrm{S}}+\sigma^{\mathrm{HC}}+\sigma^{\overline{\mathrm{HC}}}$. 


\subsubsection{Total QCD NLO cross section}

After combining all the contributions that are mentioned before, the UV, IR singularities in our final total cross section

$$
\begin{aligned}
\sigma^{\mathrm{NLO}} & =\sigma^{\mathrm{LO}}+\sigma^{\mathrm{V}}+\sigma^{\mathrm{R}} \\
& =\sigma^{\mathrm{LO}}+\sigma^{\mathrm{V}}+\sigma^{\mathrm{S}}+\sigma^{\mathrm{HC}}+\sigma^{\overline{\mathrm{HC}}}
\end{aligned}
$$

are exactly cancelled. The logarithmic dependence on the arbitrary small cutoff parameters $\delta_{s}$ and $\delta_{c}$ are then cancelled (but power-like terms survive). These cancelations can be verified numerically in our numerical calculations. The final results of the total QCD NLO cross section in the 5FNS scheme can be expressed as:

$$
\begin{aligned}
& \sigma^{\mathrm{NLO}}\left(\mathrm{pp} \rightarrow \mathrm{p} \gamma \mathrm{p} \rightarrow \mathrm{pW}^{-} \mathrm{t}+\mathrm{Y}\right) \\
&=\int_{\frac{\mathrm{M}_{\mathrm{inv}}}{\sqrt{\mathrm{s}}}}^{\sqrt{\xi_{\max }}} 2 \mathrm{zdz} \int_{\operatorname{Max}\left(\mathrm{z}^{2}, \xi_{\min }\right)}^{\xi_{\max }} \frac{\mathrm{dx}_{1}}{\mathrm{x}_{1}} \mathrm{f}_{\gamma / \mathrm{P}_{\mathrm{A}}}\left(\mathrm{x}_{1}\right)\left\{\mathrm { G } _ { \mathrm { b } / \mathrm { P } _ { \mathrm { B } } } ( \frac { \mathrm { z } ^ { 2 } } { \mathrm { x } _ { 1 } } , \mu _ { \mathrm { f } } ) \left[\hat{\sigma}^{\mathrm{LO}}+\hat{\sigma}^{\mathrm{V}}+\left(\mathrm{F}^{\mathrm{soft}}+\mathrm{F}^{\mathrm{hc}}\right) \hat{\sigma}^{\mathrm{LO}}\right.\right. \\
&\left.\left.+\left(\mathrm{F}^{1}+\mathrm{F}^{2}\right) \hat{\sigma}^{\mathrm{LO}}+\hat{\sigma}_{\mathrm{g}}^{\overline{\mathrm{HC}}}\right]+\mathrm{G}_{\mathrm{g} / \mathrm{P}_{\mathrm{B}}}\left(\frac{\mathrm{z}^{2}}{\mathrm{x}_{1}}, \mu_{\mathrm{f}}\right) \hat{\sigma}_{\overline{\mathrm{b}}}^{\mathrm{HC}}\right\}+(A \leftrightarrow B) .
\end{aligned}
$$

Here $\mathrm{F}^{\mathrm{soft}}$ and $\mathrm{F}^{\mathrm{hc}}$ are the factors contain soft and collinear singularities as well as finite terms. $\mathrm{F}^{1,2}$ are the factors that finite. In the massless $\mathrm{b}$ quark assumption, there analytical expression are

$$
\mathrm{F}^{\mathrm{soft}}+\mathrm{F}^{\mathrm{hc}}=\mathrm{C}_{\mathrm{F}} \frac{\alpha_{\mathrm{s}}}{2 \pi}\left(\frac{\mathrm{A}_{2}}{\epsilon^{2}}+\frac{\mathrm{A}_{1}}{\epsilon}+\mathrm{A}_{0}\right)
$$

with

$$
\begin{aligned}
\mathrm{A}_{2}= & 1 \\
\mathrm{~A}_{1}= & \ln \frac{\mu_{\mathrm{r}}^{2}}{\hat{\mathrm{s}}}-\ln \frac{\left(\mathrm{e}_{4}-\mathrm{e}_{4 \mathrm{z}}\right)^{2}}{\mathrm{e}_{4}^{2}-\mathrm{e}_{4 \mathrm{z}}^{2}-\mathrm{e}_{4 \mathrm{x}}^{2}}+\frac{5}{2} \\
\mathrm{~A}_{0}= & \frac{1}{2} \ln ^{2} \frac{\mu_{\mathrm{r}}^{2}}{\hat{\mathrm{s}}}-2 \ln \delta_{\mathrm{s}} \ln \frac{\mu_{\mathrm{r}}^{2}}{\hat{\mathrm{s}}}+2 \ln ^{2} \delta_{\mathrm{s}}-2 \ln \delta_{\mathrm{s}}+\ln \frac{\mu_{\mathrm{r}}^{2}}{\hat{\mathrm{s}}}-\ln \frac{\mu_{\mathrm{r}}^{2}}{\hat{\mathrm{s}}} \ln \frac{\left(\mathrm{e}_{4}-\mathrm{e}_{4 \mathrm{z}}\right)^{2}}{\mathrm{e}_{4}^{2}-\mathrm{e}_{4 \mathrm{z}}^{2}-\mathrm{e}_{4 \mathrm{x}}^{2}} \\
& +2 \ln \delta_{\mathrm{s}} \ln \frac{\left(\mathrm{e}_{4}-\mathrm{e}_{4 \mathrm{z}}\right)^{2}}{\mathrm{e}_{4}^{2}-\mathrm{e}_{4 \mathrm{z}}^{2}-\mathrm{e}_{4 \mathrm{x}}^{2}}+\frac{\mathrm{e}_{4}}{\sqrt{\mathrm{e}_{4 \mathrm{z}}^{2}+\mathrm{e}_{4 \mathrm{x}}^{2}}} \ln \frac{\mathrm{e}_{4}+\sqrt{\mathrm{e}_{4 \mathrm{z}}^{2}+\mathrm{e}_{4 \mathrm{x}}^{2}}}{\mathrm{e}_{4}-\sqrt{\mathrm{e}_{4 \mathrm{z}}^{2}+\mathrm{e}_{4 \mathrm{x}}^{2}}} \\
& +\ln ^{2} \frac{\mathrm{e}_{4}-\sqrt{\mathrm{e}_{4 \mathrm{z}}^{2}+\mathrm{e}_{4 \mathrm{x}}^{2}}}{\mathrm{e}_{4}-\mathrm{e}_{4 \mathrm{z}}}-\frac{1}{2} \ln ^{2} \frac{\mathrm{e}_{4}+\sqrt{\mathrm{e}_{4 \mathrm{z}}^{2}+\mathrm{e}_{4 \mathrm{x}}^{2}}}{\mathrm{e}_{4}-\sqrt{\mathrm{e}_{4 \mathrm{z}}^{2}+\mathrm{e}_{4 \mathrm{x}}^{2}}} \\
& +2 \mathrm{Li}_{2}\left(\frac{\mathrm{e}_{4 \mathrm{z}}-\sqrt{\mathrm{e}_{4 \mathrm{z}}^{2}+\mathrm{e}_{4 \mathrm{x}}^{2}}}{\mathrm{e}_{4}-\sqrt{\mathrm{e}_{4 \mathrm{z}}^{2}+\mathrm{e}_{4 \mathrm{x}}^{2}}}\right)-2 \mathrm{Li}_{2}\left(\frac{-\mathrm{e}_{4 \mathrm{z}}-\sqrt{\mathrm{e}_{4 \mathrm{z}}^{2}+\mathrm{e}_{4 \mathrm{x}}^{2}}}{\mathrm{e}_{4}-\mathrm{e}_{4 \mathrm{z}}}\right)
\end{aligned}
$$

and

$$
\begin{aligned}
& \mathrm{F}^{1}=\frac{\alpha_{\mathrm{s}}}{2 \pi} \int_{\frac{\mathrm{z}^{2}}{\mathrm{x}_{1}}}^{1-\delta_{\mathrm{s}}} \frac{\mathrm{dy}}{\mathrm{y}} \mathrm{G}_{\mathrm{b} / \mathrm{P}_{\mathrm{B}}}\left(\frac{\mathrm{z}^{2}}{\mathrm{x}_{1} \mathrm{y}}, \mu_{\mathrm{f}}\right)\left[\mathrm{C}_{\mathrm{F}} \frac{1+\mathrm{y}^{2}}{1-\mathrm{y}} \ln \left(\delta_{\mathrm{s}} \frac{1-\mathrm{y}}{\mathrm{y}}\right) \frac{\hat{\mathrm{s}}}{\mu_{\mathrm{f}}^{2}}+(1-\mathrm{y})\right] \\
& \mathrm{F}^{2}=\frac{\alpha_{\mathrm{s}}}{2 \pi} \int_{\frac{\mathrm{z}^{2}}{\mathrm{x}_{1}}}^{1} \frac{\mathrm{dy}}{\mathrm{y}} \mathrm{G}_{\mathrm{g} / \mathrm{P}_{\mathrm{B}}}\left(\frac{\mathrm{z}^{2}}{\mathrm{x}_{1} \mathrm{y}}, \mu_{\mathrm{f}}\right)\left[-\frac{1}{2}\left(\mathrm{y}^{2}+(1-\mathrm{y})^{2}\right) \ln \left(\delta_{\mathrm{s}} \frac{1-\mathrm{y}}{\mathrm{y}}\right) \frac{\hat{\mathrm{s}}}{\mu_{\mathrm{f}}^{2}}+\mathrm{y}(1-\mathrm{y})\right] .
\end{aligned}
$$


Notations of $\mathrm{e}_{\mathrm{i} / \mathrm{ix}}$ can be found in eq. (2.8). The dilogarithm function $\mathrm{Li}_{2}(\mathrm{x})$ is defined in ref. [106]. Technical details can be found in refs. [91, 107].

\section{$3 \quad$ Numerical results}

\subsection{Input parameters}

We take the input parameters as $\mathrm{M}_{\mathrm{p}}=0.938272046 \mathrm{GeV},\left.\alpha_{\mathrm{ew}}\left(\mathrm{m}_{\mathrm{Z}}^{2}\right)^{-1}\right|_{\overline{\mathrm{MS}}}=127.918, \mathrm{~m}_{\mathrm{Z}}=$ $91.1876 \mathrm{GeV}, \mathrm{m}_{\mathrm{W}}=80.385 \mathrm{GeV}[108]$ and we have $\sin ^{2} \theta_{\mathrm{W}}=1-\left(\mathrm{m}_{\mathrm{W}} / \mathrm{m}_{\mathrm{Z}}\right)^{2}=0.222897$. The PDFs are taken from the LHAPDF package [109]. We adopt the CTEQ6L1 and CTEQ6M PDFs $[110,111]$ for the LO and QCD higher order calculations, separately. The strong coupling constant $\alpha_{\mathrm{S}}(\mu)$ is determined by the QCD parameter $\Lambda_{5}^{\mathrm{LO}}=165 \mathrm{MeV}$ for the CTEQ6L1 and $\Lambda_{5}^{\overline{\mathrm{MS}}}=226 \mathrm{MeV}$ for the CTEQ6M, respectively. For simplicity we set the factorization scale and the renormalization scale being equal (i.e., $\mu=\mu_{\mathrm{f}}=\mu_{\mathrm{r}}$ ) and take $\mu=\mu_{0}=\left(\mathrm{m}_{\mathrm{t}}+\mathrm{m}_{\mathrm{W}}\right) / 2$ in default unless otherwise stated. Throughout this paper, we set the quark masses as $\mathrm{m}_{\mathrm{u}}=\mathrm{m}_{\mathrm{d}}=\mathrm{m}_{\mathrm{c}}=\mathrm{m}_{\mathrm{s}}=\mathrm{m}_{\mathrm{b}}=0$, The top quark pole mass is set to be $\mathrm{m}_{\mathrm{t}}=173.5 \mathrm{GeV}$. The colliding energy in the proton-proton center-of-mass system is assumed to be $\sqrt{\mathrm{s}}=14 \mathrm{TeV}$ at future LHC. We adopt BASES $[112,113]$ to do the phase space integration. The CKM matrix elements are set as unit. The decay of the top quark is expected to be dominated by the two-body channel $\mathrm{t} \rightarrow \mathrm{W}^{-} \mathrm{b}$ and the total decay width of the top quark is approximately equal to the decay width of $t \rightarrow \mathrm{W}^{-} \mathrm{b}$. Neglecting terms of order $\mathrm{m}_{\mathrm{b}}^{2} / \mathrm{m}_{\mathrm{t}}^{2}, \alpha_{\mathrm{s}}$, and $\left(\alpha_{\mathrm{s}} / \pi\right) \mathrm{m}_{\mathrm{W}}^{2} / \mathrm{m}_{\mathrm{t}}^{2}$, the width predicted in the SM at NLO is:

$$
\Gamma_{\mathrm{t}}=\frac{\alpha_{\mathrm{ew}} \mathrm{m}_{\mathrm{t}}^{3}}{16 \mathrm{~m}_{\mathrm{W}}^{2} \mathrm{~s}_{\mathrm{W}}^{2}}\left(1-\frac{\mathrm{m}_{\mathrm{W}}^{2}}{\mathrm{~m}_{\mathrm{t}}^{2}}\right)^{2}\left(1+\frac{2 \mathrm{~m}_{\mathrm{W}}^{2}}{\mathrm{~m}_{\mathrm{t}}^{2}}\right)\left[1-\frac{2 \alpha_{\mathrm{s}}}{3 \pi}\left(\frac{2 \pi^{2}}{3}-\frac{5}{2}\right)\right] .
$$

By taking $\left.\alpha_{\text {ew }}\left(\mathrm{m}_{\mathrm{Z}}^{2}\right)^{-1}\right|_{\overline{\mathrm{MS}}}=127.918$ and $\alpha_{\mathrm{s}}\left(\mathrm{m}_{\mathrm{t}}^{2}\right)=0.1079$, we obtain $\Gamma_{\mathrm{t}}=1.41595 \mathrm{GeV}$. Based on the forward proton detectors to be installed by the CMS-TOTEM and the ATLAS collaborations we choose the detected acceptances to be

- CMS-TOTEM forward detectors with $0.0015<\xi_{1}<0.5$

- CMS-TOTEM forward detectors with $0.1<\xi_{2}<0.5$

- AFP-ATLAS forward detectors with $0.0015<\xi_{3}<0.15$

which we simply refer to $\xi_{1}, \xi_{2}$ and $\xi_{3}$, respectively. During calculation we use $\xi_{1}$ in default unless otherwise stated. Note here we do not consider the decay of the heavy final states as well as the survival probability in the $\gamma \mathrm{p}$ collision or simply taken to be unit.

\subsection{Cross checks}

Before presenting the numerical predictions, several cross checks should be done.

- First, during the calculation of the tensor one loop integrals, we use our modified LoopTools and cross check with OneLoop [96] and QCDLoop [97] packages. We can get exactly the same results in each phase space point. 


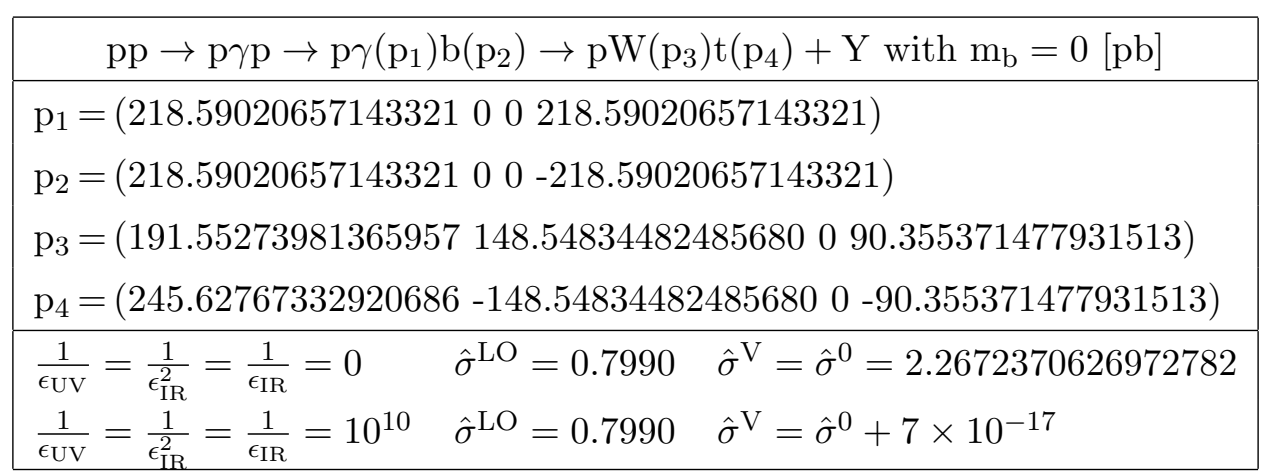

Table 1. The UV and IR divergence cancelation at one given random phase space point for the loop contribution for $\mathrm{pp} \rightarrow \mathrm{p} \gamma \mathrm{p} \rightarrow \mathrm{p} \gamma\left(\mathrm{p}_{1}\right) \mathrm{b}\left(\mathrm{p}_{2}\right) \rightarrow \mathrm{pW}\left(\mathrm{p}_{3}\right) \mathrm{t}\left(\mathrm{p}_{4}\right)+\mathrm{Y}$ with $\mathrm{m}_{\mathrm{b}}=0$.

- Second, when do the phase space integration we use BASES [112, 113] and cross check independently with Kaleu [114] especially for the hard emission contributions. We can get the same integrated results within the error.

- Third, the UV and IR safeties should be verified numerically after combining all the contributions at the NLO QCD loop level. To check this, we display enough random phase space point as well as the cancellation for different divergent parameters, see in table 1 corresponding to 5FNS massless b quark scheme. One thing that should be emphasized is $\hat{\sigma}^{\mathrm{V}}$ should include the counter-term contributions as well as the soft and collinear singularity terms coming from the real emissions. We implement this into our monte carlo codes which provide an automatic check of the dependence on these divergence parameters. We can see the UV and IR divergence can be canceled at high precision level in all the phase space thus leading the continuance of our following calculation.

- Fourth, since the total cross section is independent of the soft cutoff $\delta_{\mathrm{s}}\left(=\Delta \mathrm{E}_{\mathrm{g}} / \mathrm{E}_{\mathrm{b}}\right.$, $\left.\mathrm{E}_{\mathrm{b}}=\sqrt{\hat{\mathrm{s}}} / 2\right)$ and the collinear cutoff $\delta_{\mathrm{c}}$, we display their values for $\mathrm{pp} \rightarrow \mathrm{p} \gamma \mathrm{p} \rightarrow \mathrm{pWt}$ versus the cutoff $\delta_{\mathrm{s}}$, where we take $\delta_{\mathrm{c}}=\delta_{\mathrm{s}} / 100$. Both $\delta_{\mathrm{s}}$ and $\delta_{\mathrm{c}}$ dependence should be checked. Some of the results are listed in table 2. The detector acceptance here is chosen to be $0.0015<\xi_{1}<0.5$. It is shown clearly that the NLO QCD correction does not depend on the arbitrarily chosen values of $\delta_{\mathrm{s}}$ and $\delta_{\mathrm{c}}$ within the calculation errors. In the further numerical calculations, we fix $\delta_{\mathrm{s}}=10^{-4}$ and $\delta_{\mathrm{c}}=\delta_{\mathrm{s}} / 100$.

\subsection{Scale dependence for different forward detector acceptances}

We present the scale- $\mu$ dependence of the LO and QCD NLO corrected cross sections for $\mathrm{pp} \rightarrow \mathrm{p} \gamma \mathrm{p} \rightarrow \mathrm{pWt}+\mathrm{Y}$ for the CMS-TOTEM forward detectors with $0.0015<\xi_{1}<0.5$ in the left panel of figure 6 . Scale- $\mu$ varies from $\mu_{0} / 8$ to $2 \mu_{0}$ with $\mu_{0}=\left(\mathrm{m}_{\mathrm{W}}+\mathrm{m}_{\mathrm{t}}\right) / 2$. In the figure, solid lines with plus sign points present the LO predictions. Its cross section varies from $0.5772 \mathrm{pb}$ to $1.2717 \mathrm{pb}$ with the scale- $\mu$ varies from $\mu_{0} / 8$ to $2 \mu_{0}$. The deviation is as large as $0.6945 \mathrm{pb}$ shows some dependence on the scale. We use dotted line with times sign to present the QCD NLO corrected cross section in the 5FNS massless b quark 

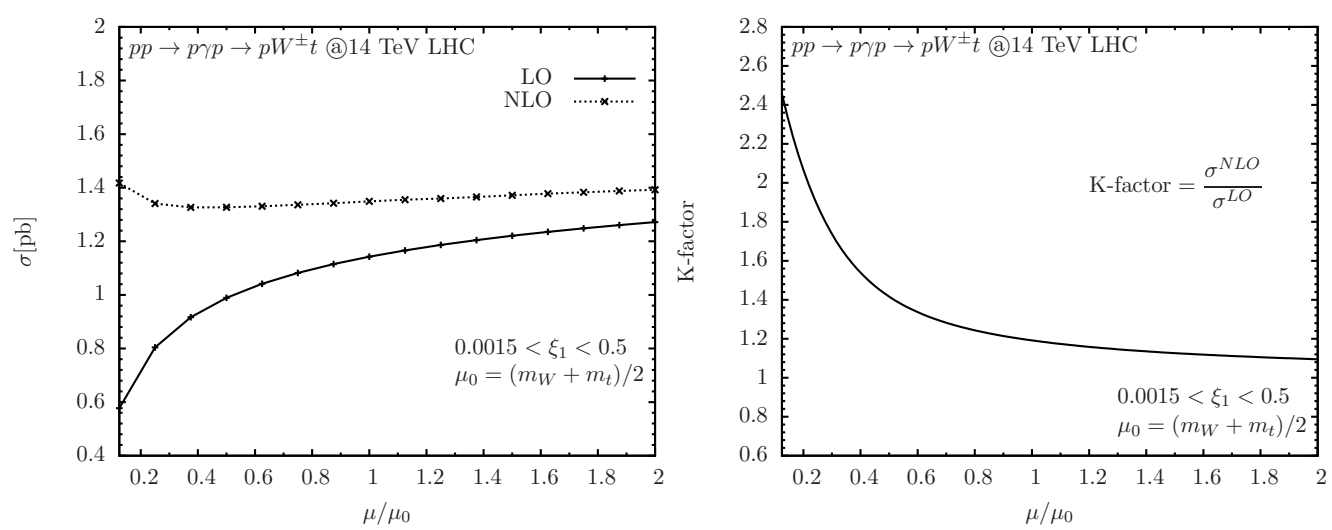

Figure 6. The scale- $\mu$ dependence of the LO and QCD NLO corrected cross sections [left panel] and $\mathrm{K}$-factor [right panel] for $\mathrm{pp} \rightarrow \mathrm{p} \gamma \mathrm{p} \rightarrow \mathrm{pWt}+\mathrm{Y}$ at the $\sqrt{\mathrm{s}}=14 \mathrm{TeV}$ LHC with $\mu_{0}=\left(\mathrm{m}_{\mathrm{W}}+\mathrm{m}_{\mathrm{t}}\right) / 2$, $\delta_{\mathrm{s}}=10^{-4}$ and $\delta_{\mathrm{c}}=\delta_{\mathrm{s}} / 100$. The experimental acceptance here is chosen to be $0.0015<\xi_{1}<0.5$ for the CMS-TOTEM forward detectors.

\begin{tabular}{|cccc|}
\hline \multicolumn{4}{|c|}{$\delta_{\mathrm{s}}$ dependence for $\sigma(\mathrm{pp} \rightarrow \mathrm{p} \gamma \mathrm{p} \rightarrow \mathrm{pWt}+\mathrm{Y})[\mathrm{pb}]$ with $\mathrm{m}_{\mathrm{b}}=0$} \\
$\delta_{\mathrm{s}}=100 \delta_{\mathrm{c}}$ & $\sigma^{\mathrm{LO}}$ & $\sigma^{\mathrm{NLO}}$ & $\Delta=\sigma^{\mathrm{NLO}}-\sigma^{\mathrm{LO}}$ \\
\hline $1 \times 10^{-3.0}$ & 1.14255073 & 1.34824330 & 0.20569257 \\
$1 \times 10^{-3.5}$ & 1.14255073 & 1.34968444 & 0.20713371 \\
$1 \times 10^{-4.0}$ & 1.14255073 & 1.34910699 & 0.20655626 \\
$1 \times 10^{-4.5}$ & 1.14255073 & 1.34921708 & 0.20666635 \\
$1 \times 10^{-5.0}$ & 1.14255073 & 1.34937836 & 0.20682763 \\
$1 \times 10^{-5.5}$ & 1.14255073 & 1.35015998 & 0.20760925 \\
$1 \times 10^{-6.0}$ & 1.14255073 & 1.34720592 & 0.20465519 \\
\hline
\end{tabular}

Table 2. The $\delta_{\mathrm{s}}$ dependence of the loop induced QCD correction to the integrated cross section for the pp $\rightarrow \mathrm{p} \gamma \mathrm{p} \rightarrow \mathrm{pWt}+\mathrm{Y}$ with $\mathrm{m}_{\mathrm{b}}=0$ at the $\sqrt{\mathrm{s}}=14 \mathrm{TeV}$ LHC where we set $\delta_{\mathrm{c}}=\delta_{\mathrm{s}} / 100$. The detector acceptance here is chosen to be $0.0015<\xi_{1}<0.5$.

scheme. The NLO cross section changes from $1.4175 \mathrm{pb}$ to $1.3922 \mathrm{pb}$ with the deviation only $0.0253 \mathrm{pb}$. We can see that if the QCD NLO corrections are taken into account, much better scale- $\mu$ independence can be obtained and the factorization/renormalization scale uncertainty can be reduced. In the right panel of figure 6 , we show the K-factor of the QCD NLO contribution as function of scale- $\mu$. K-factor is defined as $\sigma^{\mathrm{NLO}} / \sigma^{\mathrm{LO}}$. We see that $\mathrm{K}$-factor is large and sensitive in the small $\mu$ range while insensitive at the large $\mu$. Typical results of the K-factor are 2.4557, 1.3415, 1.1808 and 1.0947 for $\mu_{0} / 8, \mu_{0} / 2, \mu_{0}$ and $2 \mu_{0}$, respectively.

In figure 7 , the scale- $\mu$ dependence of the LO cross section, QCD NLO corrected cross section and K-factor are depicted in the left and right panel for the CMS-TOTEM forward detectors with $0.1<\xi_{2}<0.5$. Same as in figure 6 , we use solid lines with plus sign points present the LO predictions and dotted line with times sign to present the QCD 

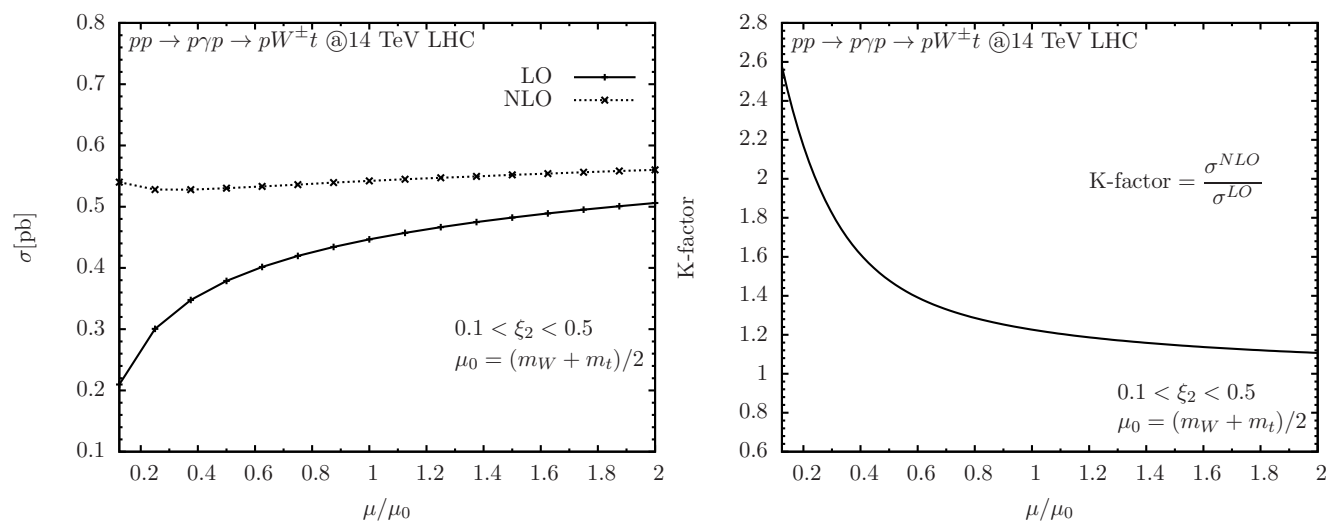

Figure 7. The scale- $\mu$ dependence of the LO and QCD NLO corrected cross sections [left panel] and $\mathrm{K}$-factor [right panel] for $\mathrm{pp} \rightarrow \mathrm{p} \gamma \mathrm{p} \rightarrow \mathrm{pWt}+\mathrm{Y}$ at the $\sqrt{\mathrm{s}}=14 \mathrm{TeV}$ LHC with $\mu_{0}=\left(\mathrm{m}_{\mathrm{W}}+\mathrm{m}_{\mathrm{t}}\right) / 2$, $\delta_{\mathrm{s}}=10^{-4}$ and $\delta_{\mathrm{c}}=\delta_{\mathrm{s}} / 100$. The experimental acceptance here is chosen to be $0.1<\xi_{2}<0.5$ for the CMS-TOTEM forward detectors.
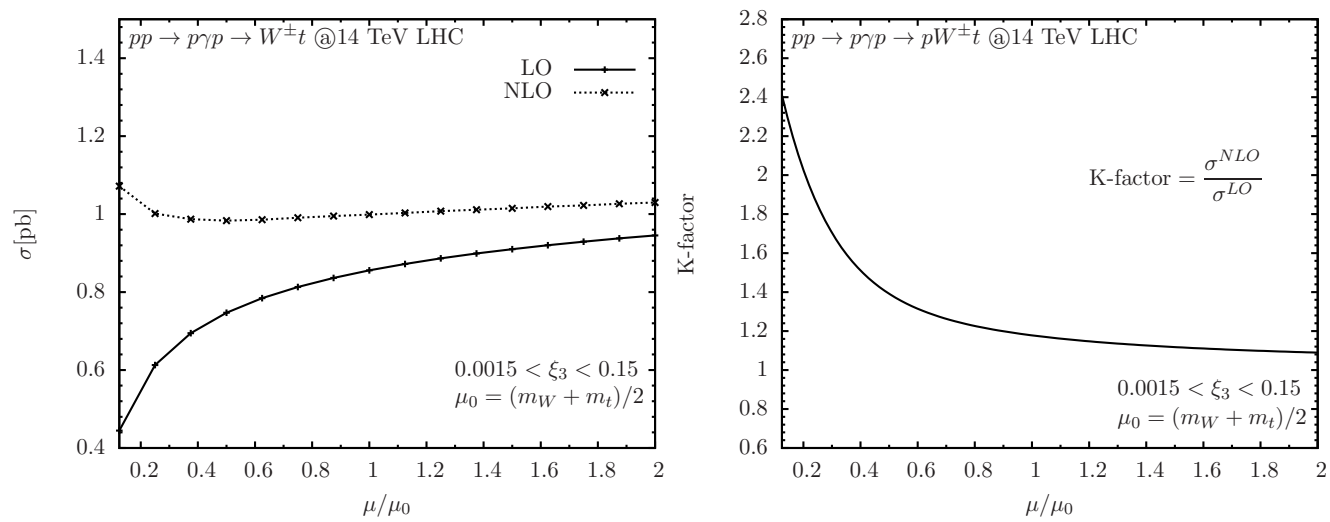

Figure 8. The scale- $\mu$ dependence of the LO and QCD NLO corrected cross sections [left panel] and $\mathrm{K}$-factor [right panel] for $\mathrm{pp} \rightarrow \mathrm{p} \gamma \mathrm{p} \rightarrow \mathrm{pWt}+\mathrm{Y}$ at the $\sqrt{\mathrm{s}}=14 \mathrm{TeV}$ LHC with $\mu_{0}=\left(\mathrm{m}_{\mathrm{W}}+\mathrm{m}_{\mathrm{t}}\right) / 2$, $\delta_{\mathrm{s}}=10^{-4}$ and $\delta_{\mathrm{c}}=\delta_{\mathrm{s}} / 100$. The experimental acceptance here is chosen to be $0.0015<\xi_{3}<0.15$ for AFP-ATLAS forward detectors.

NLO corrected cross section, respectively. When $\mu$ varies from $\mu_{0} / 8$ to $2 \mu_{0}$, their cross sections change from $0.2097(0.5485) \mathrm{pb}$ to $0.5401(0.5601) \mathrm{pb}$ with their ratio equal 2.58 (1.02). Still we can see the NLO predictions can reduce the factorization/renormalization scale uncertainty corresponding to the LO prediction. We see NLO correction shows much better scale independence through the whole range $\left[\mu_{0} / 8,2 \mu_{0}\right]$. Compare the results with $0.0015<\xi_{1}<0.5$, we see for $0.1<\xi_{2}<0.5$, in the whole range, both the LO and QCD NLO corrected cross sections are smaller, less than half of than that of $0.0015<\xi_{1}<0.5$.

For the AFP-ATLAS forward detectors with $0.0015<\xi_{3}<0.15$, the cross section for the LO and QCD NLO prediction are close to that of $0.0015<\xi_{1}<0.5$, see figure 8 . Behavior of the cross sections and K-factor on the scale- $\mu$ dependence are the same. In this case their cross sections change from 0.4447 (1.0717) pb to $0.9454(1.0297)$ pb with their ratio equal $2.13(0.96)$ when $\mu$ varies from $\mu_{0} / 8$ to $2 \mu_{0}$. We conclude again that the QCD NLO corrections can reduce the factorization/renormalization scale uncertainty. Finally 


\begin{tabular}{|ccccc|}
\hline \multicolumn{5}{|c|}{ K-factor for $\mathrm{pp} \rightarrow \mathrm{p} \gamma \mathrm{p} \rightarrow \mathrm{pWt}+\mathrm{Y}$} \\
\hline$\xi \backslash \mu$ & $\mu_{0} / 8$ & $\mu_{0} / 2$ & $\mu_{0}$ & $2 \mu_{0}$ \\
\hline$\xi_{1}$ & 2.4557 & 1.3415 & 1.1808 & 1.0947 \\
$\xi_{2}$ & 2.5761 & 1.3997 & 1.2139 & 1.1069 \\
$\xi_{3}$ & 2.4101 & 1.3166 & 1.1673 & 1.0892 \\
\hline
\end{tabular}

Table 3. The K-factor for typical value of $\mu$ for different forward detector acceptances $0.0015<$ $\xi_{1}<0.5,0.1<\xi_{2}<0.5$ and $0.0015<\xi_{3}<0.15$ with $\mu_{0}=\left(\mathrm{m}_{\mathrm{W}}+\mathrm{m}_{\mathrm{t}}\right) / 2$.

we summary the K-factor for typical value of $\mu$ in table 3 for different forward detector acceptances. In our further calculations we fix $\mu=\mu_{0}=\left(\mathrm{m}_{\mathrm{W}}+\mathrm{m}_{\mathrm{t}}\right) / 2$.

\subsection{Distribution and cross section}

In figure 9, we show the LO (solid curves) and NLO (dotted curves) transverse momentum $\left(\mathrm{p}_{\mathrm{T}}\right)$ distribution of $\mathrm{W}$ [left panel] and K-factor [right panel] for the process $\mathrm{pp} \rightarrow \mathrm{p} \gamma \mathrm{p} \rightarrow \mathrm{pWt}+\mathrm{Y}$. (a), (b) and (c) for the experimental detector acceptances $\xi_{1}$, $\xi_{2}$ and $\xi_{3}$, respectively. Of course the distributions depend on the detector acceptances, i.e., in the most efficient case $0.0015<\xi_{1}<0.5$. However, their line behaviors are the same for different detector acceptances. For the distributions of $\mathrm{p}_{\mathrm{T}}^{\text {top }}$, their behaviors are very similar to those of $\mathrm{p}_{\mathrm{T}}^{\mathrm{W}}$ thus not shown. The results show that QCD NLO contribution can enhance the LO distribution in the whole $\mathrm{p}_{\mathrm{T}}$ range. Typical K-factor is in the range of $[1.1-1.4]$.

Rapidity distributions for the $\mathrm{W}$ boson and top quark have been presented in figure 10 and figure 11. As can be seen the NLO corrections can shift the LO rapidity but in different ways for both $\mathrm{W}$ boson and top quark. For the $\mathrm{W}$ boson the distribution $\mathrm{y}^{\mathrm{W}}$, QCD NLO corrections shift the LO peak range into different $y$ and enhance them. For the top quark rapidity distributions there is not much enhancement can be found, instead, the rapidity values where they peaked shifts. Same behaviors but different values can be found for the other choices of $\xi_{2}$ and $\xi_{3}$ as can be see in figures 10(a)-10(c) and figures 11(a)-11(c), respectively. Their corresponding K-factors are present in the right panels. The reduction can be found for the K-factor when $\mathrm{y}^{\mathrm{W}}$ increase from -4 to -1 . With $\mathrm{y}^{\mathrm{W}}<-0.8 \mathrm{NLO}$ correction enhance the $\mathrm{LO}$ predictions while reverse in the range $\mathrm{y}^{\mathrm{W}} \geq-0.8$. This behavior is the same for all three value of forward detector acceptances $\xi_{1}, \xi_{2}$ and $\xi_{3}$. For $\mathrm{y}^{\text {top }}$, this value is around 0.2. That is to say, for $\mathrm{y}^{\text {top }}<0.2 \mathrm{NLO}$ correction enhance the LO predictions while reverse in the range $\mathrm{y}^{\mathrm{top}} \geq 0.2$.

In figure 12 we fix $\xi_{\min }=0.0015$ and take $\xi_{\max }$ as a running parameter from 0.15 to 1 . The LO and NLO cross sections as well as the the K-factor defined as $\sigma^{\mathrm{NLO}} / \sigma^{\mathrm{LO}}$ are presented as functions of different values of $\xi_{\max }$. The dotted, dashed and solid lines correspond to the LO, NLO predictions and K-factor, respectively. We can find that in the range $0.0015<\xi_{\max }<0.5$, both LO and NLO predictions rely on the detector acceptances while in the region $\xi_{\max }>0.5$, little contributions will shift the LO and NLO cross sections. No matter how the detector acceptances changes, the ratio of $\sigma^{\mathrm{NLO}}$ to $\sigma^{\mathrm{LO}}$ does not change 

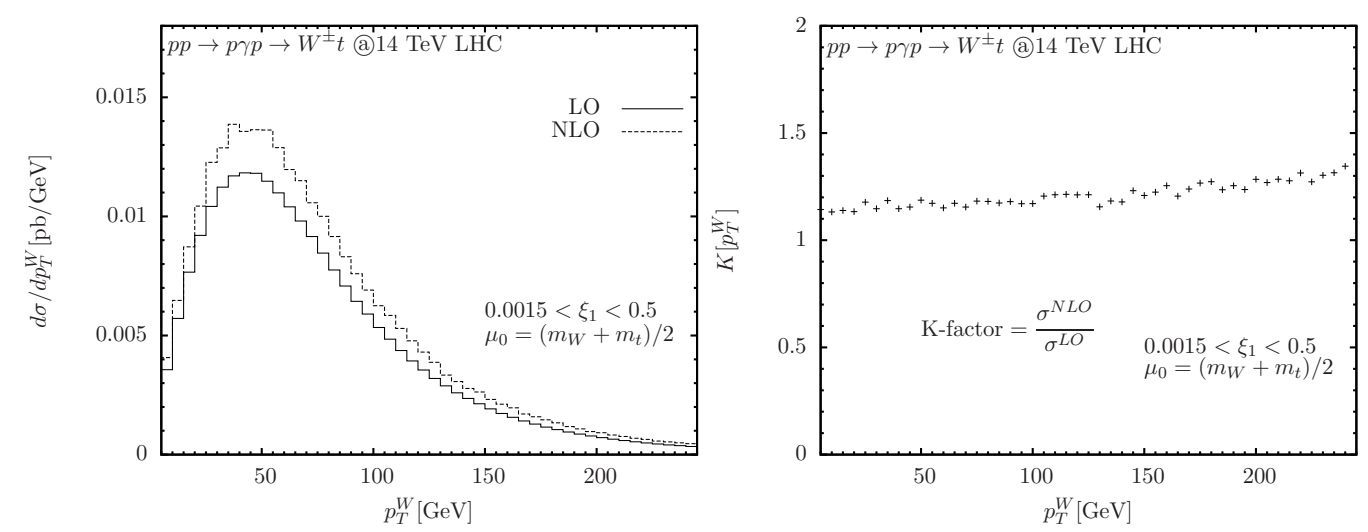

(a) $0.0015<\xi_{1}<0.5$.
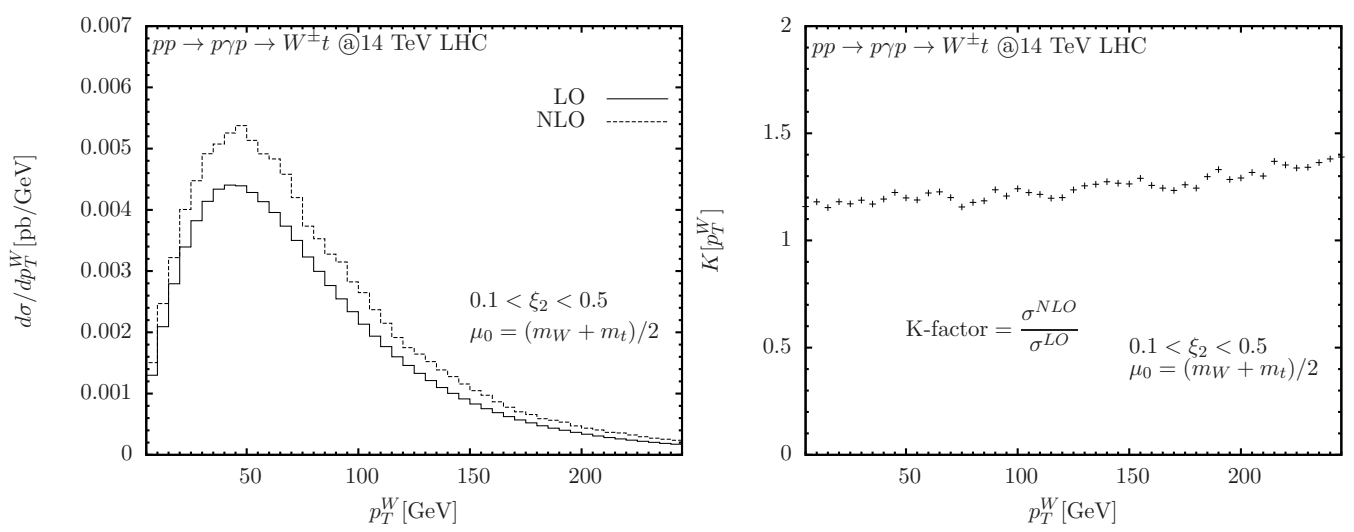

(b) $0.1<\xi_{2}<0.5$.
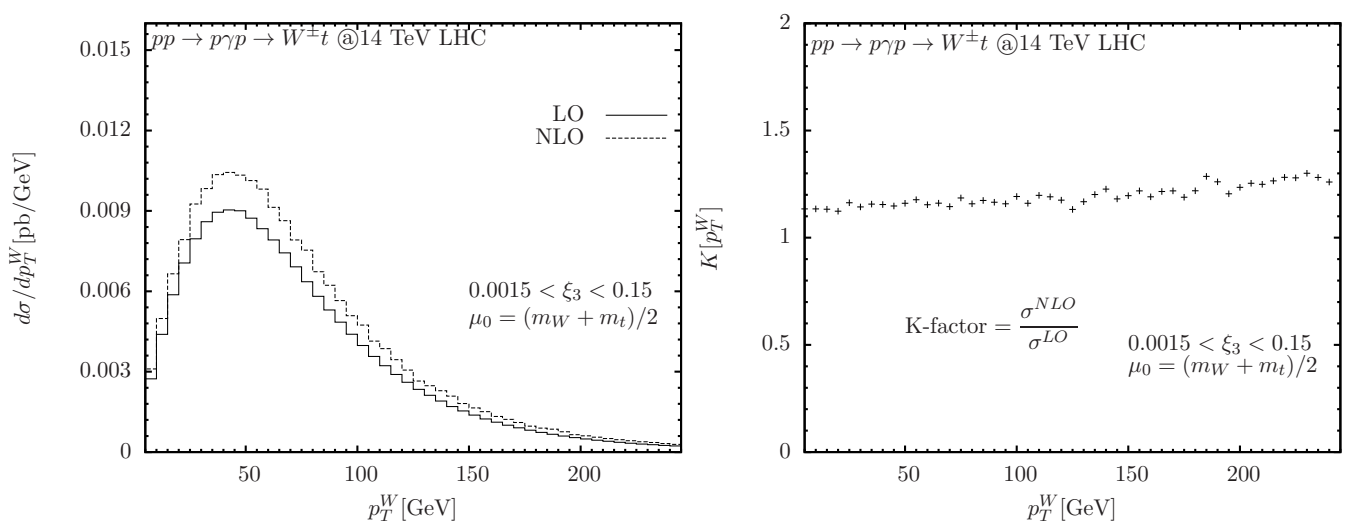

(c) $0.0015<\xi_{3}<0.15$.

Figure 9. The LO (solid curves) and NLO (dotted curves) transverse momentum $\left(p_{T}\right)$ distribution of W [left panel] and K-factor [right panel] for the process $\mathrm{pp} \rightarrow \mathrm{p} \gamma \mathrm{p} \rightarrow \mathrm{pWt}+\mathrm{Y}$ at $14 \mathrm{TeV} \mathrm{LHC}$ with $\mu=\mu_{0}=\left(\mathrm{m}_{\mathrm{W}}+\mathrm{m}_{\mathrm{t}}\right) / 2, \delta_{\mathrm{s}}=10^{-4}$ and $\delta_{\mathrm{c}}=\delta_{\mathrm{s}} / 100$. The experimental detector acceptances are $0.0015<\xi_{1}<0.5$ (a), $0.1<\xi_{2}<0.5$ (b), $0.0015<\xi_{3}<0.15$ (c). 

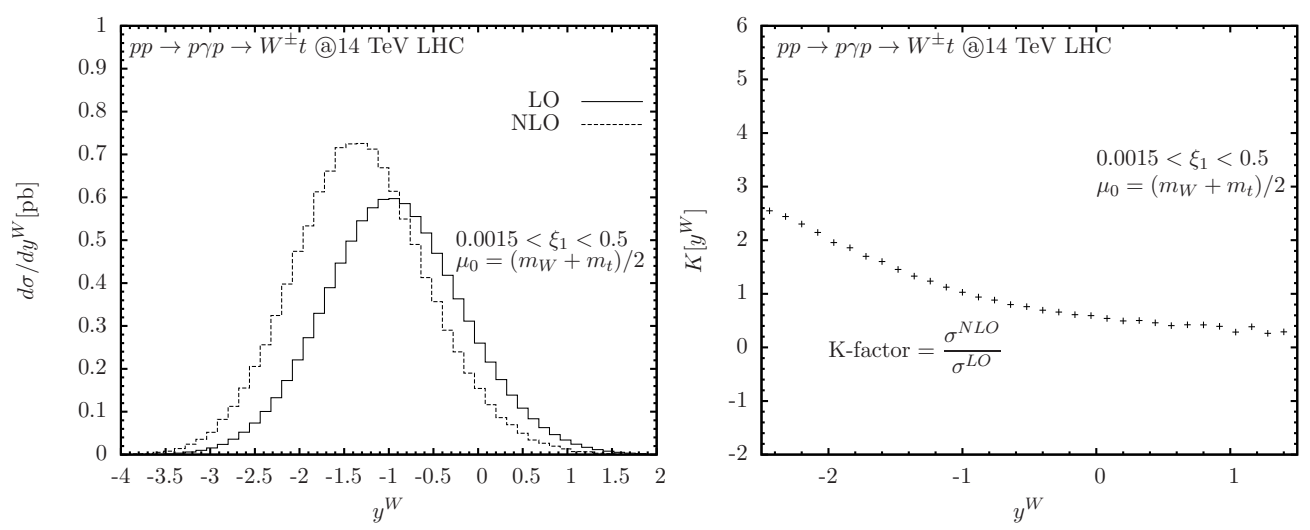

(a) $0.0015<\xi_{1}<0.5$.
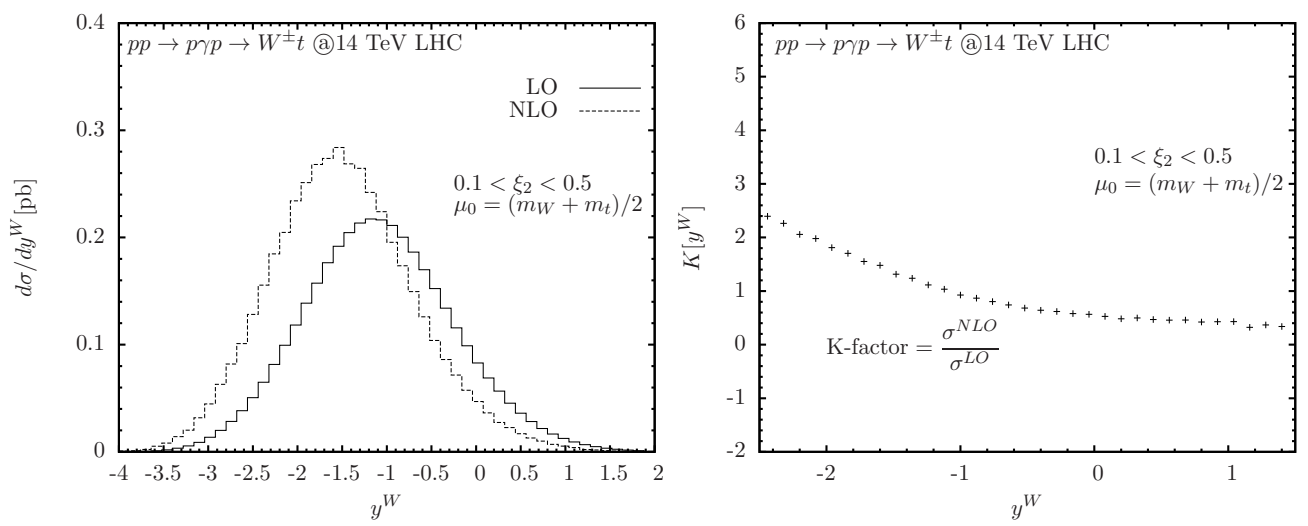

(b) $0.1<\xi_{2}<0.5$.
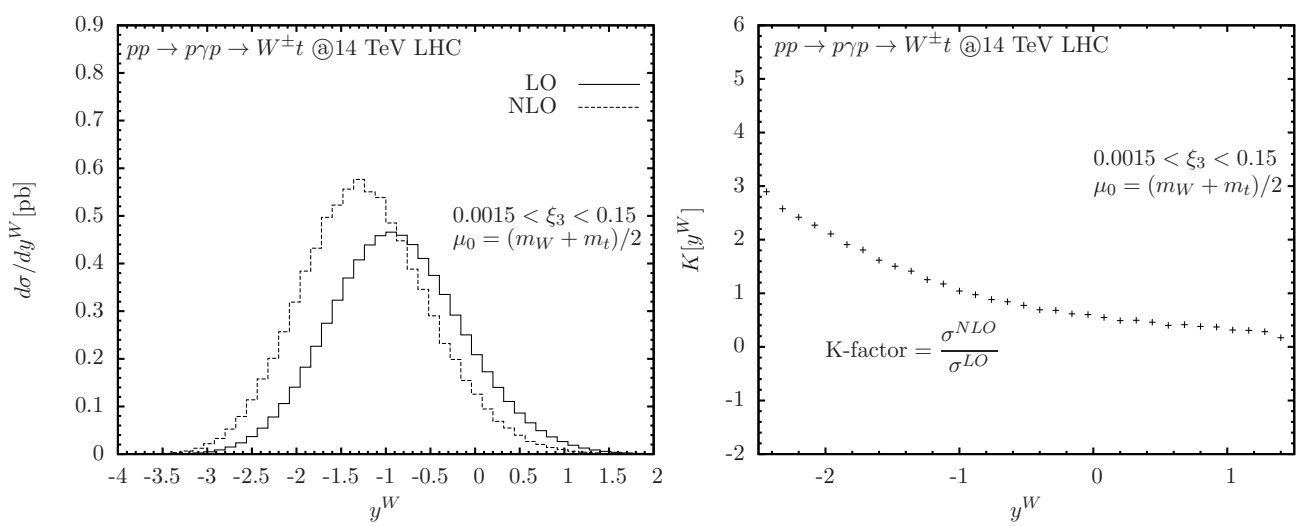

(c) $0.0015<\xi_{3}<0.15$.

Figure 10. The LO (solid curves) and NLO (dotted curves) Rapidity (y) distribution of W left panel] and K-factor [right panel] for the process pp $\rightarrow \mathrm{p} \gamma \mathrm{p} \rightarrow \mathrm{pWt}+\mathrm{Y}$ at $14 \mathrm{TeV}$ LHC with $\mu=\mu_{0}=\left(\mathrm{m}_{\mathrm{W}}+\mathrm{m}_{\mathrm{t}}\right) / 2, \delta_{\mathrm{s}}=10^{-4}$ and $\delta_{\mathrm{c}}=\delta_{\mathrm{s}} / 100$. The experimental detector acceptances are $0.0015<\xi_{1}<0.5$ (a), $0.1<\xi_{2}<0.5$ (b), $0.0015<\xi_{3}<0.15$ (c). 

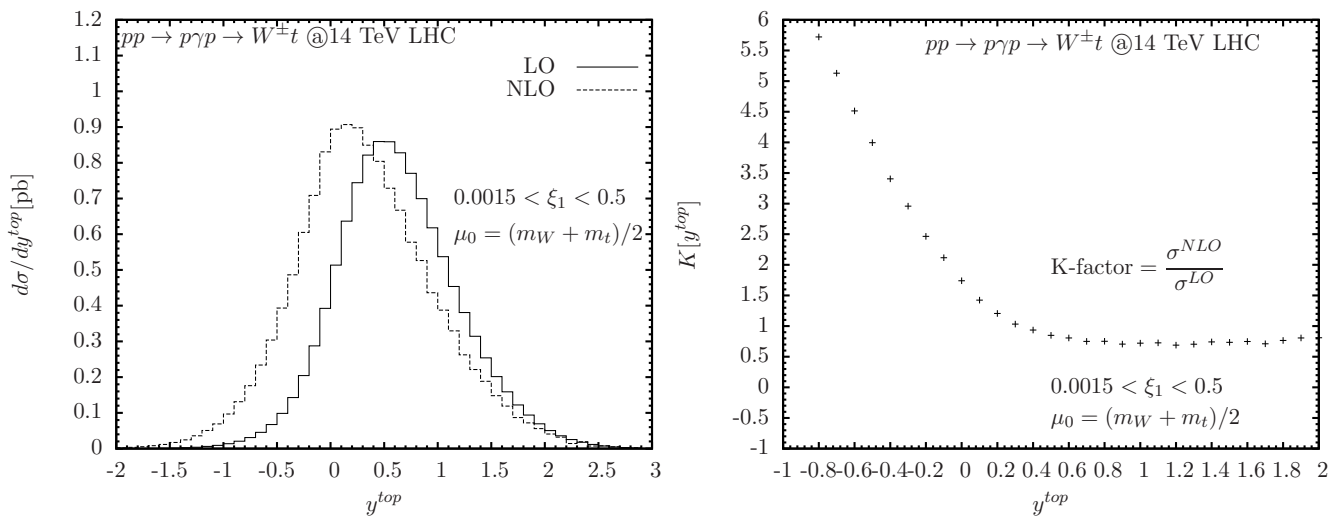

(a) $0.0015<\xi_{1}<0.5$.
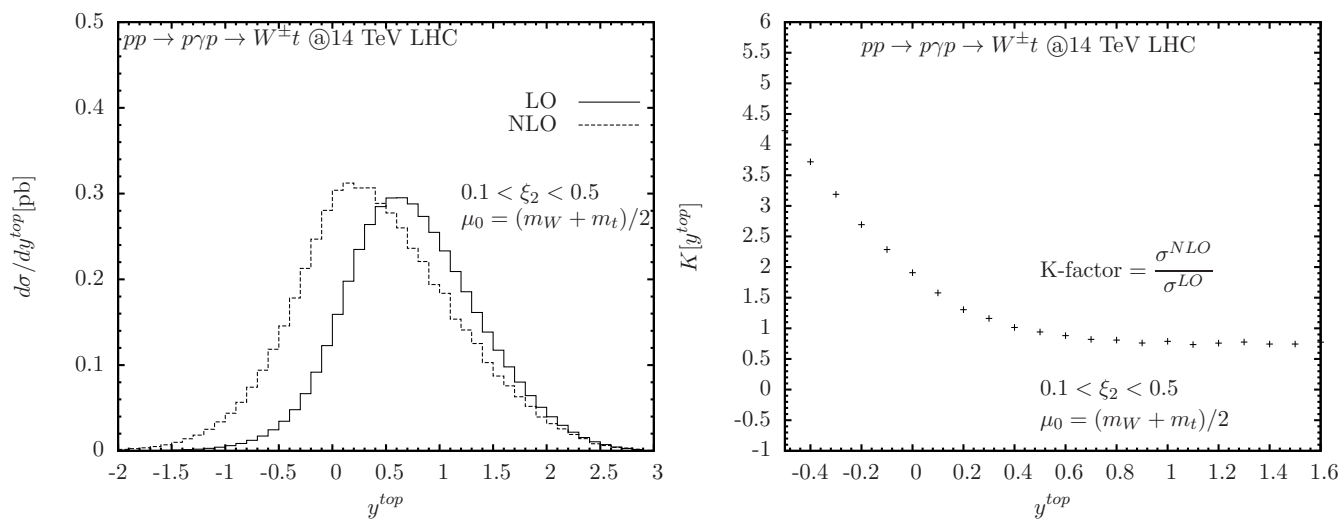

(b) $0.1<\xi_{2}<0.5$.
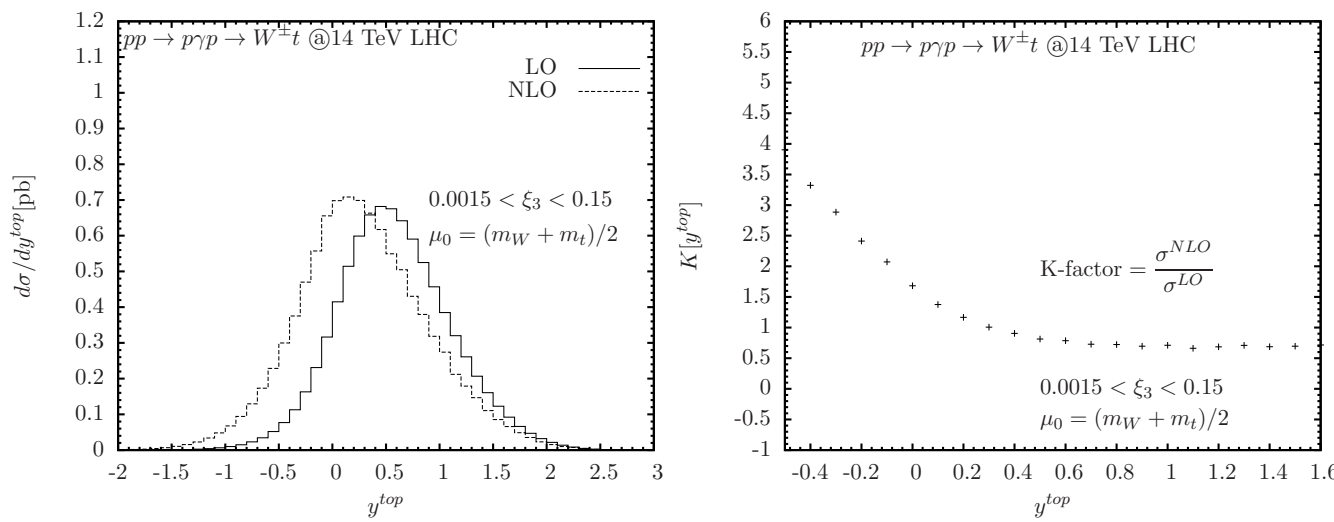

(c) $0.0015<\xi_{3}<0.15$.

Figure 11. The LO (solid curves) and NLO (dotted curves) Rapidity (y) distribution of top [left panel] and K-factor [right panel] for the process pp $\rightarrow \mathrm{p} \gamma \mathrm{p} \rightarrow \mathrm{pWt}+\mathrm{Y}$ at $14 \mathrm{TeV}$ LHC with $\mu=\mu_{0}=\left(\mathrm{m}_{\mathrm{W}}+\mathrm{m}_{\mathrm{t}}\right) / 2, \delta_{\mathrm{s}}=10^{-4}$ and $\delta_{\mathrm{c}}=\delta_{\mathrm{s}} / 100$. The experimental detector acceptances are $0.0015<\xi_{1}<0.5$ (a), $0.1<\xi_{2}<0.5$ (b), $0.0015<\xi_{3}<0.15$ (c). 


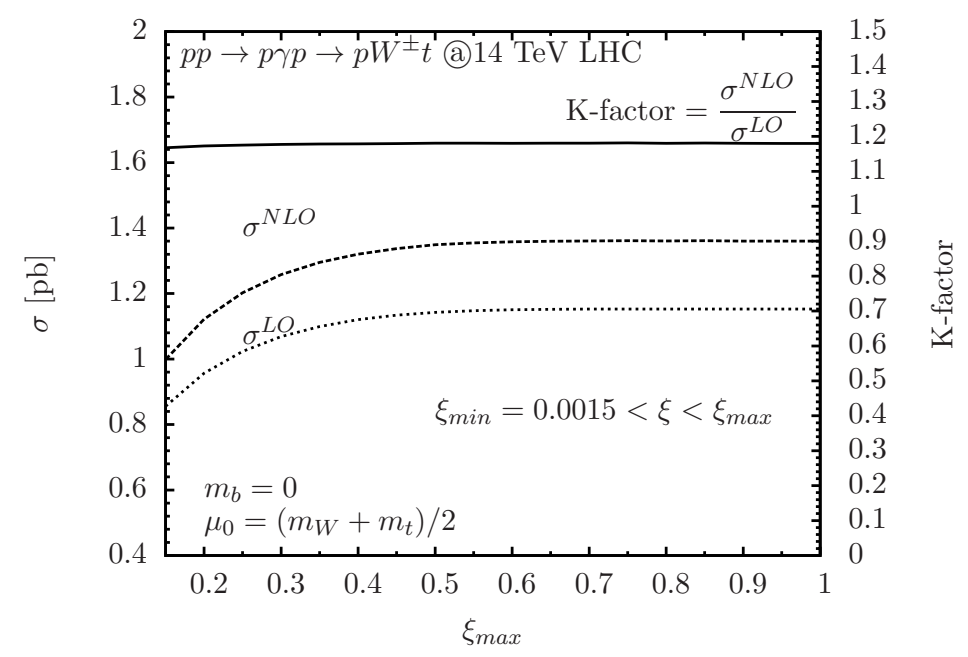

Figure 12. Cross sections for LO and NLO predictions for $\mathrm{pp} \rightarrow \mathrm{p} \gamma \mathrm{p} \rightarrow \mathrm{pWt}+\mathrm{Y}$ as well as the the K-factor as functions of different values of $\xi_{\max }$ at $14 \mathrm{TeV}$ LHC. Here we fix $\xi_{\min }=0.0015$ and take $\xi_{\max }$ as a running parameter from 0.15 to 1 . The dotted, dashed and the solid line correspond to the LO, NLO predictions and K-factor, respectively. $\mu=\mu_{0}=\left(\mathrm{m}_{\mathrm{W}}+\mathrm{m}_{\mathrm{t}}\right) / 2, \delta_{\mathrm{s}}=10^{-4}$ and $\delta_{\mathrm{c}}=\delta_{\mathrm{s}} / 100$.

much where a typical value of $\mathrm{K}$-factor equal 1.1808 in the massless assumption, leading the QCD NLO corrections up to $18.08 \%$ related to the LO predictions with our chosen parameters.

\section{Summary}

In this work, we present the precise production of Single Top and W boson associated photoproduction up to NLO QCD level through the main reaction $\mathrm{pp} \rightarrow \mathrm{p} \gamma \mathrm{p} \rightarrow \mathrm{pW}^{ \pm} \mathrm{t}+\mathrm{Y}$ at the future $14 \mathrm{TeV}$ Large Hadron Collider (LHC) for the first time, assuming a typical LHC multipurpose forward detector. We use the Five-Flavor-Number Schemes (5FNS) through the whole calculation while treat the initial state b quark as massless. This is the most important two body final state Single Top production channel at the $\gamma$ p collision. By detecting this process we can certainly in analyses aiming at top quark electrical charge, top quark mass prediction, and the CKM matrix element $\left|V_{t b}\right|$ and give complementary information for normal pp collisions. In this paper, we have employed equivalent photon approximation (EPA) for the incoming photon beams and performed detailed analysis for various forward detector acceptances $(\xi)$. We analyse their impacts on both the total cross section, renormalization/factorization scale $\mu$ dependence and some key distributions. Our results show that: QCD NLO corrections can reduce the factorization and renormalization scale uncertainty correspond to their LO predictions. They can enhance the transverse momentum $\left(\mathrm{p}_{\mathrm{T}}^{\mathrm{W}^{ \pm}}\right.$,top $)$distributions and shift the LO predictions in different ways for $\mathrm{y}^{\mathrm{W}^{ \pm}}$ and $\mathrm{y}^{\text {top }}$, leading some interesting behaviors and the crucial importance of considering the QCD NLO corrections. The typical QCD K-factor value in massless b quark scheme are 1.1808 for CMS-TOTEM forward detectors with $0.0015<\xi_{1}<0.5,1.2139$ for CMS- 
TOTEM forward detectors with $0.1<\xi_{2}<0.5$ and 1.1673 for AFP-ATLAS forward detectors with $0.0015<\xi_{3}<0.15$, respectively, with our chosen parameters.

\section{Acknowledgments}

Sun Hao thanks Dr. Inanc Sahin for his kindness to provide invaluable comments, thanks Dr. RenYou Zhang for his strong support and thanks Dr. ChongXing Yue, Lei Guo, ShaoMing Wang for useful discussions. Project supported by the National Natural Science Foundation of China (Grant No. 11205070, 11105083, 11035003, 11375104, 11105036), by Shandong Province Natural Science Foundation (Grant No. ZR2012AQ017), and by the Fundamental Research Funds for the Central Universities (No. DUT13RC(3)30).

Open Access. This article is distributed under the terms of the Creative Commons Attribution License (CC-BY 4.0), which permits any use, distribution and reproduction in any medium, provided the original author(s) and source are credited.

\section{References}

[1] ATLAS collaboration, Combination of Higgs Boson Searches with up to $4.9 \mathrm{fb}^{-1}$ of $\mathrm{pp}$ Collisions Data Taken at a center-of-mass energy of $7 \mathrm{TeV}$ with the ATLAS Experiment at the LHC, ATLAS-CONF-2011-163 (2011) [INSPIRE].

[2] CMS Collaboration, Combination of SM Higgs Searches, CMS-PAS-HIG-11-032 [INSPIRE].

[3] CDF collaboration, A. Abulencia et al., Observation of Exclusive Electron-Positron Production in Hadron-Hadron Collisions, Phys. Rev. Lett. 98 (2007) 112001 [hep-ex/0611040] [inSPIRE].

[4] CDF collaboration, T. Aaltonen et al., Search for exclusive $Z$ boson production and observation of high mass $p \bar{p} \rightarrow \gamma \gamma \rightarrow p+\ell \ell+\bar{p}$ events in $p \bar{p}$ collisions at $\sqrt{s}=1.96 \mathrm{TeV}$, Phys. Rev. Lett. 102 (2009) 222002 [arXiv:0902.2816] [InSPIRE].

[5] CDF collaboration, T. Aaltonen et al., Search for exclusive $\gamma \gamma$ production in hadron-hadron collisions, Phys. Rev. Lett. 99 (2007) 242002 [arXiv:0707.2374] [INSPIRE].

[6] CDF collaboration, T. Aaltonen et al., Observation of Exclusive Gamma Gamma Production in p p Collisions at $\sqrt{s}=1.96$ TeV, Phys. Rev. Lett. 108 (2012) 081801 [arXiv:1112.0858] [INSPIRE].

[7] CDF collaboration, T. Aaltonen et al., Observation of Exclusive Dijet Production at the

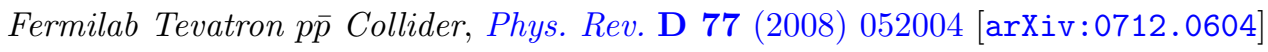
[INSPIRE].

[8] CDF collaboration, T. Aaltonen et al., Observation of exclusive charmonium production and $\gamma+\gamma$ to $\mu^{+} \mu^{-}$in p p $\bar{p}$ collisions at $\sqrt{s}=1.96$ TeV, Phys. Rev. Lett. 102 (2009) 242001 [arXiv:0902.1271] [INSPIRE].

[9] M. Tasevsky, Diffraction physics program in ATLAS experiment, ATL-PHYS-CONF-2008-019 (2008) [ATL-COM-PHYS-2008-087] [Nucl. Phys. B Proc. Suppl. 179-180 (2008) 187] [INSPIRE].

[10] M. Tasevsky, Measuring Central Exclusive Processes at LHC, arXiv:0910.5205 [InSPIRE]. 
[11] RP220 collaboration, C. Royon, Project to install roman pot detectors at $220 \mathrm{~m}$ in ATLAS, arXiv:0706.1796 [INSPIRE].

[12] B.E. Cox et al., FP420 : An RE D Proposal to Investigate the Feasibility of Installing Proton Tagging Detectors in the $420 \mathrm{~m}$ Region of the LHC, CERN-LHCC-2005-025 (2005).

[13] B.E. Cox, The FP420 R and D project at the LHC, hep-ph/0609209 [INSPIRE].

[14] M. Tasevsky, Review of Central Exclusive Production of the Higgs Boson Beyond the Standard Model, Int. J. Mod. Phys. A 29 (2014) 1446012 [arXiv:1407.8332] [InSPIRE].

[15] C. Royon, The ATLAS Forward Physics Project, arXiv:1302.0623 [InSPIRE].

[16] ATLAS collaboration, R. Staszewski, The AFP Project, Acta Phys. Polon. B 42 (2011) 1615 [arXiv: 1104.1858] [INSPIRE].

[17] J. de Favereau de Jeneret et al., High energy photon interactions at the LHC, arXiv:0908.2020 [INSPIRE].

[18] T.M.P. Tait, $t W^{-}$mode of single top quark production, Phys. Rev. D 61 (1999) 034001 [hep-ph/9909352] [INSPIRE].

[19] J. Alwall et al., Is $V_{t b} \simeq 1$ ?, Eur. Phys. J. C 49 (2007) 791 [hep-ph/0607115] [INSPIRE].

[20] J.A. Aguilar-Saavedra and A. Onofre, Using single top rapidity to measure $V_{t d}, V_{t s}, V_{t b}$ at hadron colliders, Phys. Rev. D 83 (2011) 073003 [arXiv:1002.4718] [InSPIRE].

[21] CMS collaboration, CMS technical design report, volume II: Physics performance, J. Phys. G 34 (2007) 995 [INSPIRE].

[22] A.S. Belyaev, E.E. Boos and L.V. Dudko, Single top quark at future hadron colliders: Complete signal and background study, Phys. Rev. D 59 (1999) 075001 [hep-ph/9806332] [INSPIRE].

[23] S. Ovyn and J. de Favereau de Jeneret, High energy single top photoproduction at the LHC, Nuovo Cim. B 123 (2008) 1126 [arXiv:0806.4841] [INSPIRE].

[24] J. de Favereau de Jeneret and S. Ovyn, Single top quark photoproduction at the LHC, Nucl. Phys. B Proc. Suppl. 179-180 (2008) 277 [arXiv:0806.4886] [InSPIRE].

[25] S. Atag, O. Cakir and B. Dilec, Anomalous Wtb coupling in ep collision, Phys. Lett. B 522 (2001) 76 [hep-ph/0107179] [INSPIRE].

[26] S. Dutta, A. Goyal, M. Kumar and B. Mellado, Measuring anomalous Wtb couplings at $e^{-} p$ collider, arXiv:1307.1688 [INSPIRE].

[27] D0 collaboration, V.M. Abazov et al., Observation of Single Top Quark Production, Phys. Rev. Lett. 103 (2009) 092001 [arXiv:0903.0850] [INSPIRE].

[28] D0 collaboration, V.M. Abazov et al., Measurement of the t-channel single top quark production cross section, Phys. Lett. B 682 (2010) 363 [arXiv:0907.4259] [INSPIRE].

[29] CDF collaboration, T. Aaltonen et al., First Observation of Electroweak Single Top Quark Production, Phys. Rev. Lett. 103 (2009) 092002 [arXiv:0903.0885] [InSPIRE].

[30] D.O. Carlson and C.P. Yuan, Studying the top quark via the W-gluon fusion process, Phys. Lett. B 306 (1993) 386 [INSPIRE].

[31] G. Mahlon and S.J. Parke, Improved spin basis for angular correlation studies in single top quark production at the Tevatron, Phys. Rev. D 55 (1997) 7249 [hep-ph/9611367] [INSPIRE]. 
[32] A.P. Heinson, A.S. Belyaev and E.E. Boos, Single top quarks at the Fermilab Tevatron, Phys. Rev. D 56 (1997) 3114 [hep-ph/9612424] [INSPIRE].

[33] G. Mahlon and S.J. Parke, Single top quark production at the LHC: Understanding spin, Phys. Lett. B 476 (2000) 323 [hep-ph/9912458] [INSPIRE].

[34] Z. Sullivan, Angular correlations in single-top-quark and $W_{j j}$ production at next-to-leading order, Phys. Rev. D 72 (2005) 094034 [hep-ph/0510224] [INSPIRE].

[35] TeV4LhC-Top and Electroweak Working Group, C.E. Gerber et al., Tevatron-for-LHC Report: Top and Electroweak Physics, FERMILAB-CONF-07-052 [arXiv: 0705.3251] [INSPIRE].

[36] B. Sahin and A.A. Billur, Anomalous $W_{t b}$ couplings in $\gamma p$ collision at the LHC, Phys. Rev. D 86 (2012) 074026 [arXiv:1210.3235] [INSPIRE].

[37] O. Kepka and C. Royon, Anomalous $W W \gamma$ coupling in photon-induced processes using forward detectors at the LHC, Phys. Rev. D 78 (2008) 073005 [arXiv:0808.0322] [INSPIRE].

[38] I. Sahin and A.A. Billur, Anomalous $W W \gamma$ couplings in $\gamma p$ collision at the LHC, Phys. Rev. D 83 (2011) 035011 [arXiv:1101.4998] [INSPIRE].

[39] C. Royon, E. Chapon and O. Kepka, Anomalous trilinear and quartic $W W \gamma, W W \gamma \gamma, Z Z \gamma$ and $Z Z \gamma \gamma$ couplings in photon induced processes at the LHC, PoS (EPS-HEP 2009) 380 [arXiv:0909.5237] [INSPIRE].

[40] I. Sahin and B. Sahin, Anomalous quartic $Z Z \gamma \gamma$ couplings in $\gamma p$ collision at the LHC, Phys. Rev. D 86 (2012) 115001 [arXiv:1211.3100] [INSPIRE].

[41] A. Senol, $Z Z \gamma$ and $Z \gamma \gamma$ anomalous couplings in $\gamma p$ collision at the LHC, Phys. Rev. D 87 (2013) 073003 [arXiv:1301.6914] [INSPIRE].

[42] A. Senol, Anomalous quartic $W W \gamma \gamma$ and $Z Z \gamma \gamma$ couplings in $\gamma p$ collision at the LHC, Int. J. Mod. Phys. A 29 (2014) 1450148 [arXiv:1311.1370] [inSPIRE].

[43] M. Köksal and S.C. İnan, Anomalous tq $\gamma$ couplings in $\gamma p$ collision at the LHC, Adv. High Energy Phys. 2014 (2014) 935840 [arXiv:1305.7096] [INSPIRE].

[44] H. Sun, Probe anomalous tq $\gamma$ couplings through single top photoproduction at the LHC, Nucl. Phys. B 886 (2014) 691 [arXiv: 1402.1817] [INSPIRE].

[45] S. Heim, Q.-H. Cao, R. Schwienhorst and C.-P. Yuan, Next-to-leading order QCD corrections to s-channel single top quark production and decay at the LHC, Phys. Rev. D 81 (2010) 034005 [arXiv:0911.0620] [INSPIRE].

[46] W.T. Giele, S. Keller and E. Laenen, QCD corrections to $W$ boson plus heavy quark production at the Tevatron, Phys. Lett. B 372 (1996) 141 [hep-ph/9511449] [INSPIRE].

[47] S. Zhu, Next-to-leading order QCD corrections to $b g \rightarrow t W^{-}$at the CERN Large Hadron Collider, Phys. Lett. B 524 (2002) 283 [Erratum ibid. B 537 ((2002)) 351] [hep-ph/0109269] [INSPIRE].

[48] Q.-H. Cao, Demonstration of One Cutoff Phase Space Slicing Method: Next-to-Leading Order QCD Corrections to the $t W$ Associated Production in Hadron Collision, arXiv:0801.1539 [INSPIRE].

[49] M. Beccaria et al., A Complete one-loop description of associated $t W$ production at LHC and a search for possible genuine supersymmetric effects, Eur. Phys. J. C 53 (2008) 257 [arXiv:0705.3101] [INSPIRE]. 
[50] S. Frixione, E. Laenen, P. Motylinski, B.R. Webber and C.D. White, Single-top hadroproduction in association with a W boson, JHEP 07 (2008) 029 [arXiv:0805.3067] [INSPIRE].

[51] J.M. Campbell and F. Tramontano, Next-to-leading order corrections to Wt production and decay, Nucl. Phys. B 726 (2005) 109 [hep-ph/0506289] [INSPIRE].

[52] F. Cascioli, S. Kallweit, P. Maierhöfer and S. Pozzorini, A unified NLO description of top-pair and associated Wt production, Eur. Phys. J. C 74 (2014) 2783 [arXiv:1312.0546] [INSPIRE].

[53] V.M. Budnev, I.F. Ginzburg, G.V. Meledin and V.G. Serbo, The Two photon particle production mechanism. Physical problems. Applications. Equivalent photon approximation, Phys. Rept. 15 (1975) 181 [INSPIRE].

[54] G. Baur, K. Hencken, D. Trautmann, S. Sadovsky and Y. Kharlov, Coherent $\gamma \gamma$ and $\gamma A$ interactions in very peripheral collisions at relativistic ion colliders, Phys. Rept. 364 (2002) 359 [hep-ph/0112211] [INSPIRE].

[55] K. Piotrzkowski, Tagging two photon production at the CERN LHC, Phys. Rev. D 63 (2001) 071502 [hep-ex/0009065] [INSPIRE].

[56] S. Ovyn, Associated $W$ and Higgs boson photoproduction and other electroweak photon induced processes at the LHC, Nucl. Phys. B Proc. Suppl. 179-180 (2008) 269 [arXiv:0806.1157] [INSPIRE].

[57] N. Schul and K. Piotrzkowski, Detection of two-photon exclusive production of supersymmetric pairs at the LHC, Nucl. Phys. B Proc. Suppl. 179-180 (2008) 289 [arXiv:0806.1097] [INSPIRE].

[58] K. Piotrzkowski and N. Schul, Two-photon exclusive production of supersymmetric pairs at the LHC, AIP Conf. Proc. 1200 (2010) 434 [arXiv:0910.0202] [InSPIRE].

[59] S. Heinemeyer, V.A. Khoze, M.G. Ryskin, W.J. Stirling, M. Tasevsky and G. Weiglein, Studying the MSSM Higgs sector by forward proton tagging at the LHC, Eur. Phys. J. C 53 (2008) 231 [arXiv:0708.3052] [INSPIRE].

[60] S. Heinemeyer, V.A. Khoze, M. Tasevsky and G. Weiglein, Exclusive Production of the MSSM Higgs Bosons at the LHC, arXiv: 1206.0183 [INSPIRE].

[61] S. Heinemeyer, V.A. Khoze, M.G. Ryskin, M. Tasevsky and G. Weiglein, BSM Higgs Physics in the Exclusive Forward Proton Mode at the LHC, Eur. Phys. J. C 71 (2011) 1649 [arXiv: 1012.5007] [INSPIRE].

[62] M. Tasevsky, Exclusive MSSM Higgs production at the LHC after Run I, Eur. Phys. J. C 73 (2013) 2672 [arXiv: 1309.7772] [INSPIRE].

[63] S. Atag, S.C. İnan and I. Sahin, Extra dimensions in photon-induced two lepton final states at the CERN-LHC, Phys. Rev. D 80 (2009) 075009 [arXiv:0904.2687] [INSPIRE].

[64] S. Atag, S.C. İnan and I. Sahin, Extra dimensions in $\gamma \gamma \rightarrow \gamma \gamma$ process at the CERN-LHC, JHEP 09 (2010) 042 [arXiv: 1005.4792] [INSPIRE].

[65] H. Sun, Large Extra Dimension effects through Light-by-Light Scattering at the CERN LHC, Eur. Phys. J. C 74 (2014) 2977 [arXiv: 1406.3897] [INSPIRE].

[66] I. Sahin et al., Probe of extra dimensions in $\gamma q \rightarrow \gamma q$ at the LHC, Phys. Rev. D 88 (2013) 095016 [arXiv: 1304.5737] [INSPIRE]. 
[67] I. Sahin and S.C. Inan, Probe of unparticles at the LHC in exclusive two lepton and two photon production via photon-photon fusion, JHEP 09 (2009) 069 [arXiv:0907.3290] [INSPIRE].

[68] H. Sun and C.-X. Yue, Precise photoproduction of the charged top-pions at the LHC with forward detector acceptances, Eur. Phys. J. C 74 (2014) 2823 [arXiv:1401.0250] [INSPIRE].

[69] T. Pierzchala and K. Piotrzkowski, Sensitivity to anomalous quartic gauge couplings in photon-photon interactions at the LHC, Nucl. Phys. B Proc. Suppl. 179-180 (2008) 257 [arXiv:0807.1121] [INSPIRE].

[70] E. Chapon, C. Royon and O. Kepka, Anomalous quartic $W W \gamma \gamma, Z Z \gamma \gamma$ and trilinear $W W \gamma$ couplings in two-photon processes at high luminosity at the LHC, Phys. Rev. D 81 (2010) 074003 [arXiv:0912.5161] [INSPIRE].

[71] R.S. Gupta, Probing Quartic Neutral Gauge Boson Couplings using diffractive photon fusion at the LHC, Phys. Rev. D 85 (2012) 014006 [arXiv:1111.3354] [InSPIRE].

[72] S. Fichet, G. von Gersdorff, O. Kepka, B. Lenzi, C. Royon and M. Saimpert, Probing new physics in diphoton production with proton tagging at the Large Hadron Collider, Phys. Rev. D 89 (2014) 114004 [arXiv:1312.5153] [INSPIRE].

[73] I. Sahin and M. Koksal, Search for electromagnetic properties of the neutrinos at the LHC, JHEP 03 (2011) 100 [arXiv: 1010.3434] [INSPIRE].

[74] S. Atag and A.A. Billur, Possibility of Determining $\tau$ Lepton Electromagnetic Moments in $\gamma \gamma \rightarrow \tau^{+} \tau^{-}$Process at the CERN-LHC, JHEP 11 (2010) 060 [arXiv:1005.2841] [INSPIRE].

[75] I. Sahin, Electromagnetic properties of the neutrinos in $\gamma p$ collision at the LHC, Phys. Rev. D 85 (2012) 033002 [arXiv:1201.4364] [INSPIRE].

[76] H. Sun, Dark matter searches in jet plus missing energy events in $\gamma p$ collisions at the CERN LHC, Phys. Rev. D 90 (2014) 035018 [arXiv: 1407.5356] [InSPIRE].

[77] M. Chaichian, P. Hoyer, K. Huitu, V.A. Khoze and A.D. Pilkington, Searching for the triplet Higgs sector via central exclusive production at the LHC, JHEP 05 (2009) 011 [arXiv:0901.3746] [INSPIRE].

[78] S. Dawson, C.B. Jackson, L. Reina and D. Wackeroth, Higgs boson production with one bottom quark jet at hadron colliders, Phys. Rev. Lett. 94 (2005) 031802 [hep-ph/0408077] [INSPIRE].

[79] S. Dittmaier, M. Krämer, M. Spira and M. Walser, Charged-Higgs-boson production at the LHC: NLO supersymmetric QCD corrections, Phys. Rev. D 83 (2011) 055005 [arXiv:0906.2648] [INSPIRE].

[80] J.M. Campbell, R. Frederix, F. Maltoni and F. Tramontano, Next-to-Leading-Order Predictions for t-Channel Single-Top Production at Hadron Colliders, Phys. Rev. Lett. 102 (2009) 182003 [arXiv:0903.0005] [INSPIRE].

[81] J.M. Campbell, F. Caola, F. Febres Cordero, L. Reina and D. Wackeroth, NLO QCD predictions for $W+1$ jet and $W+2$ jet production with at least one $b$ jet at the $7 \mathrm{TeV} \mathrm{LHC}$, Phys. Rev. D 86 (2012) 034021 [arXiv:1107.3714] [INSPIRE].

[82] R. Harlander, M. Krämer and M. Schumacher, Bottom-quark associated Higgs-boson production: reconciling the four- and five-flavour scheme approach, CERN-PH-TH-2011-134 [arXiv:1112.3478] [INSPIRE]. 
[83] M. Worek, NLO mass effects in $b \bar{b} b \bar{b}$ production at the LHC, PoS (RADCOR 2013) 038 [arXiv:1311.2396] [INSPIRE].

[84] H.B. Hartanto and L. Reina, Hard-photon production with b jets at hadron colliders, Phys. Rev. D 89 (2014) 074001 [arXiv: 1312.2384] [INSPIRE].

[85] F. Maltoni, G. Ridolfi and M. Ubiali, b-initiated processes at the LHC: a reappraisal, JHEP 07 (2012) 022 [Erratum ibid. 1304 (2013) 095] [arXiv: 1203.6393] [INSPIRE].

[86] J.M. Campbell et al., Associated Production of a $W$ Boson and One b Jet, Phys. Rev. D 79 (2009) 034023 [arXiv:0809.3003] [INSPIRE].

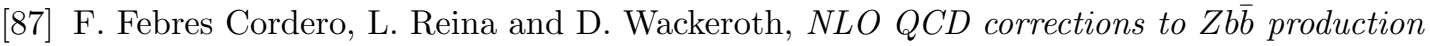
with massive bottom quarks at the Fermilab Tevatron, Phys. Rev. D 78 (2008) 074014 [arXiv:0806.0808] [INSPIRE].

[88] F. Febres Cordero, L. Reina and D. Wackeroth, NLO QCD corrections to $W$ boson

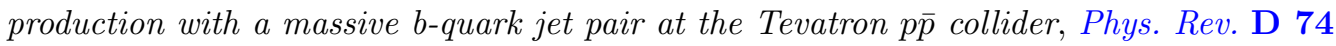
(2006) 034007 [hep-ph/0606102] [INSPIRE].

[89] F. Febres Cordero, L. Reina and D. Wackeroth, W- and Z-boson production with a massive bottom-quark pair at the Large Hadron Collider, Phys. Rev. D 80 (2009) 034015 [arXiv: 0906.1923] [INSPIRE].

[90] F. Febres Cordero, L. Reina and D. Wackeroth, Associated Production of a $W$ or $Z$ Boson with Bottom Quarks at the Tevatron and the LHC, PoS (RADCOR2009) 055 [arXiv: 1001.3362] [INSPIRE].

[91] B.W. Harris and J.F. Owens, The Two cutoff phase space slicing method, Phys. Rev. D 65 (2002) 094032 [hep-ph/0102128] [INSPIRE].

[92] T. Hahn, Generating Feynman diagrams and amplitudes with FeynArts 3, Comput. Phys. Commun. 140 (2001) 418 [hep-ph/0012260] [INSPIRE].

[93] T. Hahn, Automatic loop calculations with FeynArts, FormCalc and LoopTools, Nucl. Phys. B Proc. Suppl. 89 (2000) 231 [hep-ph/0005029] [INSPIRE].

[94] S. Agrawal, T. Hahn and E. Mirabella, FormCalc 7, J. Phys. Conf. Ser. 368 (2012) 012054 [arXiv:1112.0124] [INSPIRE].

[95] T. Hahn and M. Pérez-Victoria, Automatized one loop calculations in four-dimensions and D-dimensions, Comput. Phys. Commun. 118 (1999) 153 [hep-ph/9807565] [INSPIRE].

[96] A. van Hameren, OneLOop: For the evaluation of one-loop scalar functions, Comput. Phys. Commun. 182 (2011) 2427 [arXiv: 1007.4716] [INSPIRE].

[97] R.K. Ellis and G. Zanderighi, Scalar one-loop integrals for QCD, JHEP 02 (2008) 002 [arXiv:0712.1851] [INSPIRE].

[98] A. Belyaev and E. Boos, Single top quark $t W+X$ production at the CERN LHC: A Closer look, Phys. Rev. D 63 (2001) 034012 [hep-ph/0003260] [INSPIRE].

[99] B.P. Kersevan and I. Hinchliffe, A Consistent prescription for the production involving massive quarks in hadron collisions, JHEP 09 (2006) 033 [hep-ph/0603068] [INSPIRE].

[100] T. Plehn and C. Weydert, Charged Higgs production with a top in MC@NLO, PoS(CHARGED 2010) 026 [arXiv: 1012.3761] [INSPIRE]. 
[101] T. Binoth, D. Goncalves Netto, D. Lopez-Val, K. Mawatari, T. Plehn and I. Wigmore, Automized Squark-Neutralino Production to Next-to-Leading Order, Phys. Rev. D 84 (2011) 075005 [arXiv: 1108.1250] [INSPIRE].

[102] R.-Y. Zhang, H. Yan, W.-G. Ma, S.-M. Wang, L. Guo and L. Han, $W_{H} / Z_{H}$ production associated with a T-odd (anti)quark at the LHC in NLO QCD, Phys. Rev. D 85 (2012) 015017 [arXiv: 1112.5225] [INSPIRE].

[103] D. Goncalves-Netto, D. Lopez-Val, K. Mawatari, T. Plehn and I. Wigmore, Sgluon Pair Production to Next-to-Leading Order, Phys. Rev. D 85 (2012) 114024 [arXiv:1203.6358] [INSPIRE].

[104] W. Hollik, J.M. Lindert and D. Pagani, NLO corrections to squark-squark production and decay at the LHC, JHEP 03 (2013) 139 [arXiv:1207.1071] [INSPIRE].

[105] B. Fuks, M. Klasen, D.R. Lamprea and M. Rothering, Gaugino production in proton-proton collisions at a center-of-mass energy of 8 TeV, JHEP 10 (2012) 081 [arXiv:1207.2159] [INSPIRE].

[106] L. Lewin, Polylogarithms and associated functions, North-Holland, Amsterdam The Netherlands (1983).

[107] W. Beenakker, H. Kuijf, W.L. van Neerven and J. Smith, QCD Corrections to Heavy Quark Production in pp Collisions, Phys. Rev. D 40 (1989) 54 [InSPIRE].

[108] Particle Data Group collaboration, J. Beringer et al., Review of Particle Physics (RPP), Phys. Rev. D 86 (2012) 010001 [InSPIRE].

[109] M.R. Whalley, D. Bourilkov and R.C. Group, The Les Houches accord PDFs (LHAPDF) and LHAGLUE, hep-ph/0508110 [INSPIRE].

[110] J. Pumplin, D.R. Stump, J. Huston, H.L. Lai, P.M. Nadolsky and W.K. Tung, New generation of parton distributions with uncertainties from global QCD analysis, JHEP $\mathbf{0 7}$ (2002) 012 [hep-ph/0201195] [INSPIRE].

[111] D. Stump et al., Inclusive jet production, parton distributions and the search for new physics, JHEP 10 (2003) 046 [hep-ph/0303013] [INSPIRE].

[112] S. Kawabata, A New version of the multidimensional integration and event generation package BASES/SPRING, Comput. Phys. Commun. 88 (1995) 309 [INSPIRE].

[113] F. Yuasa, D. Perret-Gallix, S. Kawabata and T. Ishikawa, PVM-GRACE, Nucl. Instrum. Meth. A 389 (1997) 77 [inSPIRE].

[114] A. van Hameren, Kaleu: A General-Purpose Parton-Level Phase Space Generator, arXiv: 1003.4953 [INSPIRE]. 